\title{
Stochastic polarized line formation
}

\section{Zeeman line transfer in a random magnetic field ${ }^{\star}$}

\author{
H. Frisch ${ }^{1}$, M. Sampoorna ${ }^{1,2, \star \star}$, and K. N. Nagendra ${ }^{1,2}$ \\ ${ }^{1}$ Laboratoire Cassiopée (CNRS, UMR 6202), Observatoire de la Côte d'Azur, BP 4229, 06304 Nice Cedex 4, France \\ e-mail: frisch@obs-nice.fr \\ 2 Indian Institute of Astrophysics, Koramangala Layout, Bangalore 560 034, India
}

Received 17 November 2005 / Accepted 27 March 2006

ABSTRACT

\begin{abstract}
Context. The Zeeman effect produced by a turbulent magnetic field or a random distribution of flux tubes is usually treated in the microturbulent or macroturbulent limits where the Zeeman propagation matrix or the Stokes parameters, respectively, are averaged over the probability distribution function of the magnetic field when computing polarized line profiles.

Aims. To overcome these simplifying assumptions we consider the Zeeman effect from a random magnetic field which has a finite correlation length that can be varied from zero to infinity and thus made comparable to the photon mean free-path.

Methods. The vector magnetic field is modeled by a Kubo-Anderson process, a piecewise constant Markov process characterized by a correlation length and a probability distribution function for the random values of the magnetic field. The micro and macro turbulent limits are recovered when the correlation goes to zero or infinity.

Results. An integral equation is constructed for the mean propagation operator and explicit expressions are obtained for the mean values and second-order moments of the Stokes parameters at the surface of a Milne-Eddington type atmosphere. The expression given by Landi Degl'Innocenti (1994) for the mean Stokes parameters is recovered. Mean values and rms fluctuations around the mean values are calculated numerically for a random magnetic field with isotropic Gaussian fluctuations. The effects of a finite correlation length are discussed in detail. Various extensions of the Milne-Eddington and magnetic field model are considered and the corresponding integral equations for the mean propagation operator are given.

Conclusions. The rms fluctuations of the Stokes parameters are shown to be very sensitive to the correlation length of the magnetic field. It is suggested to use them as a diagnostic tool to determine the scale of unresolved features in the solar atmosphere.
\end{abstract}

Key words. line: formation - polarization - magnetic fields - methods: analytical - Sun: atmosphere

\section{Introduction}

The Zeeman effect has been used in Astrophysics for more than a century to measure magnetic fields in the Sun, stars and other objects. The very first analyses of the Zeeman effect were carried out with uniform magnetic fields. Together with a higher quality of data, appeared multi-components models (Stenflo 1994), each component having a different but uniform, or slowly varying, magnetic field. For these models, the observable Stokes parameters are given by a conveniently weighted average of the Stokes parameters of each component. Prompted by measurements of asymmetrical Stokes $V$ profiles, multi-components models of another type were introduced under the name of MISMA (Sánchez Almeida et al. 1996). In this model, each component is optically thin and the Zeeman propagation matrix is replaced by an average over the various components. These two types of models can be made quite sophisticated. With the terminology used for random velocity fields broadening, one can say that the first model is of the macroturbulent type, since the averaging is over the radiation field, whereas the second type of model is of the microturbulent type since the averaging is done locally over the propagation matrix.

\footnotetext{
* Appendix A is only available in electronic form at http://www. edpsciences.org

$\star \star$ JAP, Dept. of Physics, IISc, Bangalore 560 012, India.
}

These two types of models may be insufficient to encompass the complexity of the solar atmosphere which shows inhomogeneities, undoubtedly related to the magnetic field structure, down to scales at the limit of the resolution power of present day telescopes. For example there is an active discussion on the fine structure of sunspot penumbrae. It seems accepted that penumbral magnetic fields have a more or less horizontal component in the form of flux tubes embedded in a more vertical background. However the diameter of these flux tubes and their spatial distribution is still a matter of controversy, the number quoted in the literature varying from $1-15 \mathrm{~km}$ to $100 \mathrm{~km}$ (Sánchez Almeida 1998; Martinez Pillet 2000; Borrero et al. 2005). In addition, because of very large kinetic and magnetic Reynolds numbers prevailing in the solar atmosphere (Childress \& Gilbert 1995), turbulent magnetic and velocity fields have spectra extending over a wide range of wave-numbers. We were thus strongly motivated to consider the Zeeman effect in a medium where the magnetic field is random with a correlation length, i.e. characteristic scale of variation, comparable to radiative transfer characteristic scales. The importance of this problem has been stressed again recently (Landi Degl'Innocenti 2003; Landi Degl'Innocenti \& Landolfi 2004, henceforth LL04).

The general regime, neither macro nor microturbulent, leads to polarized radiative transfer equations with random coefficients. Only a few papers have been devoted to this subject in the past (see however, Faulstich 1980; Landi Degl'Innocenti 1994, 
henceforth L94). Recently this field seems to be receiving some renewed interest (Caroll \& Staude 2003, 2005; Silant'ev 2005). Similar problems, somewhat simpler though, have been solved in the seventies for the transfer of unpolarized radiation in the presence of a turbulent velocity with a finite correlation length (see Mihalas 1978, for a list of references). Turbulent velocity field models introduced were less or more sophisticated. The simplest one, is the Kubo-Anderson process (henceforth KAP). For radiative transfer problems, it was employed in the context of turbulent velocity fields for LTE lines (Auvergne et al. 1973) and non-LTE lines (Frisch \& Frisch 1976; Froeschlé \& Frisch 1980), and also in the context of random magnetic fields for the Zeeman (L94) and Hanle (Frisch 2006) effects. Actually the KAP was introduced for nuclear magnetic resonance (Anderson 1954; Kubo 1954). It was also employed to model the electric field in the stochastic Stark effect (Brissaud \& Frisch 1971; Frisch \& Brissaud 1971). The name Kubo-Anderson process was introduced in Auvergne et al. (1973).

The idea of the KAP is to describe the atmosphere in a number of "eddies" having lengths distributed according to a Poisson distribution with given density. It is assumed that in each eddy the magnetic field and other random parameters, such as the velocity or temperature, are constant and their values drawn at random from a probability distribution function. The mean polarized radiation field is obtained by averaging over this distribution and the distribution of the length of the eddies. A KAP is thus characterized by a correlation length and a distribution function for the values of the random variables. The correlation length and the distribution function can be selected independently. This model is fairly simple but has the correct micro and macroturbulent limits corresponding to a correlation length which is zero or infinite. As we show here, when associated to a simple atmospheric model like the Milne-Eddington model, it yields a convolution-type integral equation for the mean propagation operator from which one can deduce explicit expressions for the mean and rms fluctuations of the Stokes parameters at the surface of the atmosphere, and also for the cross-correlations between Stokes parameters. In L94, only the mean Stokes parameters at the surface are considered. It is quite clear that having explicit expressions is very useful for exploring finite correlation length effects.

In this paper the main focus is on the effects of random magnetic fields with a finite correlation length. For a full description of say, turbulent eddies or random distribution of flux tubes in a sunspot penumbrae, it is necessary to incorporate all the other relevant atmospheric parameters which typically should be described by the same type of random process as the magnetic field, in particular the same correlation length. When the magnetic field is described by a KAP, incorporating other random parameters, in particular a velocity field, also described by a KAP with the same correlation length as the magnetic field is no additional work as we explain in the Remark at the end of Sect. 2.6.

In Sect. 2 we define the random magnetic field model, establish a convolution-type integral equation for the mean propagation operator, solve it exactly for its Laplace transform and give an explicit expression for the mean value of the Stokes parameters at the surface of the atmosphere. The latter is used in Sect. 3 to study numerically the sensitivity of the mean Stokes parameters to the correlation length of a random magnetic field with isotropic Gaussian fluctuations. In Sect. 4 we establish an explicit expression for the second-order moments of the Stokes parameters and study numerically the dispersion of the Stokes parameters about their mean values. The second-order moments give also access to the mean cross-correlations between Stokes parameters. In Sect. 5 we introduce various extensions of the Milne-Eddington and magnetic field model and establish the corresponding integral equations for the mean propagation operator. A summary of the main results is presented in Sect. 6.

\section{The mean Stokes parameters}

\subsection{The surface value of the Stokes parameters}

We consider a line formed in LTE in semi-infinite onedimensional medium and assume that the source function is a linear function of depth. The radiative transfer equation for the Stokes vector $\boldsymbol{I}=[I, Q, U, V]^{\mathrm{T}}$ for rays propagating outwards along the normal to the surface may be written as

$\frac{\mathrm{d}}{\mathrm{d} s} \boldsymbol{I}(s)=\boldsymbol{K}(s)[\boldsymbol{I}(s)-\boldsymbol{S}(s)]$

Here, $s$ is the ray-path coordinate which varies inside the medium from 0 to $\infty$, with the surface at $s=0, K$ the $4 \times 4$ propagation matrix and $S(s)$ the source function vector which is of the form

$\boldsymbol{S}(s)=\left(B_{0}+B_{1} s\right) \boldsymbol{U}$,

where $B_{0}$ and $B_{1}$ are constants and $\boldsymbol{U}$ a constant vector. If $\boldsymbol{S}(s)$ is of thermal origin, say the Planck function, $\boldsymbol{U}=[1,0,0,0]^{\mathrm{T}}$. The Stokes vector and the matrix $\boldsymbol{K}$ are functions of frequency. We omit the frequency variable since there is no scattering term to couple the frequencies of incident and emergent beams.

Following the usual procedure, we define the evolution operator $\boldsymbol{O}\left(s, s^{\prime}\right)$, as the linear operator which transforms $\boldsymbol{I}\left(s^{\prime}\right)$ into $\boldsymbol{I}(s)$ when the source term $\boldsymbol{S}$ in Eq. (1) vanishes (Landi Degl'Innocenti 1987; see also the appendix A). Since photons propagate from positive $s$ (inside) to $s=0$ (surface), we always take $s^{\prime}>s$. The formal solution of the transfer equation at $s=0$ may be written as

$\boldsymbol{I}(0)=\left[B_{0} \boldsymbol{E}+B_{1} \int_{0}^{\infty} \boldsymbol{O}(0, s) \mathrm{d} s\right] \boldsymbol{U}$

where $\boldsymbol{E}$ is the $4 \times 4$ identity matrix. We are interested in the calculation of $\langle\boldsymbol{I}(0)\rangle_{\mathrm{KA}}$, the mean value of $\boldsymbol{I}(0)$ over all the realizations of the random magnetic field, given by

$\langle\boldsymbol{I}(0)\rangle_{\mathrm{KA}}=\left[B_{0} \boldsymbol{E}+B_{1} \int_{0}^{\infty}\langle\boldsymbol{O}(0, s)\rangle_{\mathrm{KA}} \mathrm{d} s\right] \boldsymbol{U}$.

The notation $\langle\cdots\rangle_{\mathrm{KA}}$ will always mean an average over all the realizations of the KAP.

\subsection{The random magnetic field model}

Assuming that the magnetic field $\boldsymbol{H}(s)$ is a KAP implies that $\boldsymbol{H}(s)$ is piecewise constant, jumping at randomly chosen points between random values. The jumping point $s_{i}$ are uniformly and independently distributed in $[0,+\infty]$ with a Poisson distribution of density $v$ independent of $s$. In each interval $s_{i-1}<$ $s<s_{i}$, the magnetic field takes a constant value $\boldsymbol{H}(s)=\boldsymbol{H}_{i}$. The $\boldsymbol{H}_{i}$ are random variables with a probability distribution function $P(\boldsymbol{H})$ independent of $s$. Hence a KAP is fully characterized by a probability distribution function and a correlation length here defined as $1 / v$. We recall that for a Poisson distribution of density $v$, the probability of having $r$ jumps in an interval of length $L$ is $\mathrm{e}^{-v L}(v L)^{r} / r$ !. Since $\boldsymbol{H}(s)$ is a KAP, any element of the Zeeman propagation matrix $\boldsymbol{K}$ is also a KAP. 
The absence of memory of the Poisson process implies that a KAP is a Markov process (see the definition after Eq. (7)). The Markov property and the fact that $\boldsymbol{H}(s)$ is piecewise constant are the two properties which allow us to obtain an integral equation for the mean propagation operator. In addition, because $P(\boldsymbol{H})$ and $v$ are chosen independent of $s$, the KAP is a stationary process (unconditioned statistical properties are invariant under space translations). As a consequence, the integral equation for the mean propagation operator is of the convolution type (see Eq. (10)). Examples of integral equations, which are not of the convolution type because the stationarity assumption has been relaxed, are given in Sect. 5.

\subsection{The mean propagation operator}

The mean value $\langle\boldsymbol{O}(0, s)\rangle_{\mathrm{KA}}$ can be calculated by summing the contributions from realizations having $N=0, N=1, N=2$, etc. jumping points (e.g. Brissaud \& Frisch 1971). This technique yields the mean value as sum of a series. The latter is equivalent to a Neumann series expansion of the convolution-type integral equation (see Eq. (10)). Following Brissaud \& Frisch (1974; see also Auvergne et al. 1973) we show how to establish a integral equation for $\langle\boldsymbol{O}(0, s)\rangle_{\mathrm{KA}}$ directly. A summation method is used in Sect. 4 to calculate the second-order moments of the Stokes parameters.

When the propagation matrix is independent of space, the propagation operator $\boldsymbol{O}\left(s, s^{\prime}\right)$ is an exponential and depends only on the difference $s-s^{\prime}$. Henceforth referred to as the "static" evolution operator and denoted by $\boldsymbol{O}_{\mathrm{S}}$ (S stands for static), it may be written as

$\boldsymbol{O}_{\mathrm{S}}\left(s, s^{\prime}\right)=\mathrm{e}^{-\left(s^{\prime}-s\right) \boldsymbol{K}}$.

The exponential of the constant matrix $\boldsymbol{K}$ is defined in a standard way, e.g. by its power-series expansion. The operator $\boldsymbol{O}_{\mathrm{S}}$ will play an important role in the following.

First we consider all the realizations without jumping point between 0 and $s$. For each realization $\boldsymbol{K}$ is constant in the interval $[0, s]$ and the propagation operator is given by its static value. The probability that there is no jump in the interval $[0, s]$ is $\mathrm{e}^{-v s}$. Thus, the contribution to the mean propagation operator from the realizations with no jump is given by:

$\langle\boldsymbol{O}(0, s)\rangle_{\text {KA }}^{\text {(no jump) }}=\mathrm{e}^{-v s}\left\langle\mathrm{e}^{-\boldsymbol{K} s}\right\rangle=\mathrm{e}^{-v s}\left\langle\boldsymbol{O}_{\mathrm{S}}(s)\right\rangle$,

where $\langle\ldots\rangle$ denotes an average involving only the probability distribution function $P(\boldsymbol{H})$ of the magnetic field.

Next we assume that there are one or several jumping points between 0 and $s$ and denote by $t$ the last jumping point before $s$. For a Poisson distribution, the probability distribution of $s-t$ is the same as the probability distribution of the intervals between successive jumps. Hence the probability that $t$ falls within the small interval $\left[s^{\prime}, s^{\prime}+\delta s^{\prime}\right]$ is given by the usual Poisson formula $v \delta s^{\prime} \mathrm{e}^{-v\left(s-s^{\prime}\right)}$.

The mean of the propagation operator, when there is at least one jump, is obtained by integrating its conditional mean, knowing that the last jump falls in the small interval $\delta s^{\prime}$, weighted by the probability of the conditioning event. The integral is over all possible values of $s^{\prime}$, that is from 0 to $s$. (Note that the probability that the KAP has its last jump in the small interval $\delta s^{\prime}$ is proportional to $\delta s^{\prime}$, but the conditional probability is, to leading order, independent of $\delta s^{\prime}$.) The mean of the propagation operator for the case with at least one jump may thus be written as

$\langle\boldsymbol{O}(0, s)\rangle_{\mathrm{KA}}^{\text {(jumps) }}=\int_{0}^{s} v \mathrm{e}^{-v\left(s-s^{\prime}\right)}\langle\boldsymbol{O}(0, s)\rangle_{\mathrm{KA}, s^{\prime}} \mathrm{d} s^{\prime}$, where $\langle\ldots\rangle_{\mathrm{KA}, \mathrm{s}^{\prime}}$ denotes the conditional mean, evaluated with the conditional probability.

Two key properties are now used: (i) the Markov property of the KAP, which guarantees that, after conditioning, the "past" $\left(0<t<s^{\prime}\right)$ and the "future" $\left(s^{\prime}<t<s\right)$ are independent and (ii) the semi-group property $\boldsymbol{O}(0, s)=\boldsymbol{O}\left(0, s^{\prime}\right) \boldsymbol{O}\left(s^{\prime}, s\right)$ (see Appendix A).

Using (i) and (ii), and the fact that the propagation operator in the interval $\left[s^{\prime}, s\right]$ is just the static one, we have

$\langle\boldsymbol{O}(0, s)\rangle_{\mathrm{KA}, s^{\prime}}=\left\langle\boldsymbol{O}\left(0, s^{\prime}\right)\right\rangle_{\mathrm{KA}, s^{\prime}}\left\langle\boldsymbol{O}_{\mathrm{S}}\left(s-s^{\prime}\right)\right\rangle$.

We claim that

$\left\langle\boldsymbol{O}\left(0, s^{\prime}\right)\right\rangle_{\mathrm{KA}, s^{\prime}}=\left\langle\boldsymbol{O}\left(0, s^{\prime}\right)\right\rangle_{\mathrm{KA}}$.

Indeed, the knowledge that a jump occurs at $s^{\prime}$, imposes no constraint on previous jumping points and previous values of $\boldsymbol{K}(t)$. Observe that the r.h.s. is an unconditional average.

Adding the contributions from Eqs. (6) and (7), we obtain a closed convolution-type integral equation for the mean propagation operator:

$$
\begin{aligned}
\langle\boldsymbol{O}(0, s)\rangle_{\mathrm{KA}}= & \mathrm{e}^{-v s}\left\langle\boldsymbol{O}_{\mathrm{S}}(s)\right\rangle \\
& +\int_{0}^{s} v \mathrm{e}^{-v\left(s-s^{\prime}\right)}\left\langle\boldsymbol{O}\left(0, s^{\prime}\right)\right\rangle_{\mathrm{KA}}\left\langle\boldsymbol{O}_{\mathrm{S}}\left(s-s^{\prime}\right)\right\rangle \mathrm{d} s^{\prime} .
\end{aligned}
$$

The stationary property implies that Eq. (10), written here for the interval $[0, s]$, holds for any interval $\left[s_{1}, s_{2}\right]$, provided the necessary changes are made.

Equation (10) can be solved explicitly by introducing the Laplace transforms,

$$
\begin{aligned}
& \tilde{\boldsymbol{O}}(0, p) \equiv \int_{0}^{\infty} \mathrm{e}^{-p s} \boldsymbol{O}(0, s) \mathrm{d} s \\
& \tilde{\boldsymbol{O}}_{\mathrm{S}}(p) \equiv \int_{0}^{\infty} \mathrm{e}^{-p s} \boldsymbol{O}_{\mathrm{S}}(s) \mathrm{d} s
\end{aligned}
$$

where it is assumed that $\mathfrak{R}(p) \geq 0$ to ensure convergence. The notation $\equiv$ means that we are introducing a definition. Equation (5) implies that

$$
\left\langle\tilde{\boldsymbol{O}}_{\mathrm{S}}(p)\right\rangle=\left\langle[p \boldsymbol{E}+\boldsymbol{K}]^{-1}\right\rangle .
$$

Taking the Laplace transform of Eq. (10) and transforming the integral $\int_{0}^{\infty} \mathrm{d} s \int_{0}^{s} \ldots \mathrm{d} s^{\prime}$ into $\int_{0}^{\infty} \mathrm{d} s^{\prime} \int_{s^{\prime}}^{\infty} \ldots \mathrm{d} s$, we obtain

$$
\langle\tilde{\boldsymbol{O}}(0, p)\rangle_{\mathrm{KA}}=\left\langle\tilde{\boldsymbol{O}}_{\mathrm{S}}(p+v)\right\rangle+v\langle\tilde{\boldsymbol{O}}(0, p)\rangle_{\mathrm{KA}}\left\langle\tilde{\boldsymbol{O}}_{\mathrm{S}}(p+v)\right\rangle,
$$

which leads to

$$
\langle\tilde{\boldsymbol{O}}(0, p)\rangle_{\mathrm{KA}}=\left\langle\tilde{\boldsymbol{O}}_{\mathrm{S}}(p+v)\right\rangle\left[\boldsymbol{E}-v\left\langle\tilde{\boldsymbol{O}}_{\mathrm{S}}(p+v)\right\rangle\right]^{-1}
$$

We note that the two factors in Eq. (15) commute, the product being of the form $\boldsymbol{A}[\boldsymbol{E}+\alpha \boldsymbol{A}]^{-1}$ with $\alpha$ a scalar. This can be shown by expanding the second factor in powers of $\boldsymbol{A}$ or by using $\boldsymbol{E}=\boldsymbol{A} \boldsymbol{A}^{-1}$.

In principle, by performing an inverse Laplace transform on the r.h.s. of Eq. (15) we can obtain the mean propagation operator $\langle\boldsymbol{O}(0, s)\rangle_{\mathrm{KA}}$. Actually in our applications, only the Laplace transform is needed. 


\subsection{Mean values of the Stokes parameters at the surface}

Returning to Eq. (4), we see that the integral in the r.h.s. is the Laplace transform of $\langle O(0, s)\rangle_{\mathrm{KA}}$ for $p=0$ (see Eq. (11)). The mean value of the Stokes vector at the surface can thus be written as

$\langle\boldsymbol{I}(0)\rangle_{\mathrm{KA}}=\left[B_{0} \boldsymbol{E}+B_{1}\langle\tilde{\boldsymbol{O}}(0,0)\rangle_{\mathrm{KA}}\right] \boldsymbol{U}$,

where, according to Eq. (15),

$\langle\tilde{\boldsymbol{O}}(0,0)\rangle_{\mathrm{KA}}=\left\langle\tilde{\boldsymbol{O}}_{\mathrm{S}}(v)\right\rangle\left[\boldsymbol{E}-v\left\langle\tilde{\boldsymbol{O}}_{\mathrm{S}}(v)\right\rangle\right]^{-1}$,

with $\left\langle\tilde{\boldsymbol{O}}_{\mathrm{S}}(v)\right\rangle$ given by Eq. (13) with $p=v$.

Equation (16), combined with Eqs. (17) and (13), yields an explicit expression for the mean value of the Stokes vector at the surface. The sole averaging which has to be performed is the averaging over $P(\boldsymbol{H})$ in Eq. (13).

As mentioned above, this expression has first been obtained in L94, with a stochastic magnetic field model identical to ours, even if it is not referred to as a KAP. The proof, which is very elegant, starts from Eq. (4). The integral over $[0, \infty]$ is first replaced by a sum from $i=1$ to $\infty$ over all the intervals $\left[s_{i-1}, s_{i}\right]$. Elementary algebra shows that each term in the sum is of the form

$\left[\prod_{j=2}^{j=i} \exp \left(-\Delta s_{j-1} \boldsymbol{K}_{j-1}\right)\right]\left[\boldsymbol{E}-\exp \left(-\Delta s_{j} \boldsymbol{K}_{j}\right)\right] \boldsymbol{K}_{j}^{-1}$,

where $\boldsymbol{K}_{j}$ is the constant value of Zeeman propagation matrix in the interval $\Delta s_{j}=s_{j}-s_{j-1}$. The $s_{i}$ are assumed to be distributed according to a Poisson law characterized by a density $1 / s_{e}$ and the $\boldsymbol{K}_{i}$ to be uncorrelated. The mean value of the Stokes vector is then obtained by averaging over all the possible partitions of the $s$-axis and over the probability distribution function of $\boldsymbol{K}$. The expression given in L94 is

$$
\begin{aligned}
& \langle\boldsymbol{I}(0)\rangle_{\mathrm{KA}}=B_{0} \boldsymbol{U}+B_{1}\left\{\left[\boldsymbol{E}-\left\langle\left(1+s_{e} \boldsymbol{K}\right)^{-1}\right\rangle\right]^{-1}\right. \\
& \left.\quad \times\left[\left\langle\boldsymbol{K}^{-1}\right\rangle-\left\langle\left(1+s_{e} \boldsymbol{K}\right)^{-1} \boldsymbol{K}^{-1}\right\rangle\right]\right\} \boldsymbol{U} .
\end{aligned}
$$

Replacing $s_{e}$ by $1 / v$, it can be checked that Eq. (19) is identical to our result. A more detailed proof can be found in LL04.

\subsection{The macro and micro-turbulent limits}

The macroturbulent limit corresponds to a correlation length $1 / v$ going to infinity. In this case the magnetic field is independent of optical depth but its value is random with a probability distribution function $P(\boldsymbol{H})$. Setting $v=0$ in Eq. (17) we obtain for the macroturbulent limit,

$\langle\boldsymbol{I}(0)\rangle_{\text {macro }}=\left[B_{0} \boldsymbol{E}+B_{1}\left\langle\boldsymbol{K}^{-1}\right\rangle\right] \boldsymbol{U}$.

In the microturbulent limit, the correlation length $1 / v$ goes to zero. Using

$\left\langle[v \boldsymbol{E}+\boldsymbol{K}]^{-1}\right\rangle \simeq \frac{1}{v}\left\langle\boldsymbol{E}-\frac{1}{v} \boldsymbol{K}\right\rangle, \quad v \rightarrow \infty$,

one obtains

$\langle\boldsymbol{I}(0)\rangle_{\text {micro }}=\left[B_{0} \boldsymbol{E}+B_{1}\langle\boldsymbol{K}\rangle^{-1}\right] \boldsymbol{U}$.
The micro and macroturbulent limits can be constructed with the standard Unno-Rachkovsky solution (e.g. Rees 1987; Jefferies et al. 1989; LL04). It suffices to average $\boldsymbol{K}$ over $P(\boldsymbol{H})$ in the microturbulent limit and the Unno-Rachkovsky solution itself in the macroturbulent limit. Following L94, we can say that the result given in Eqs. (16) and (17) is a generalization of the traditional Unno-Rachkovsky solution for random magnetic fields. We can also remark that the macroturbulent limit is of the same nature as a standard multi-component model whereas the microturbulent limit is of the MISMA type. Of course, these models usually incorporate many physical processes in addition to the Zeeman effect.

\subsection{Residual emergent Stokes vector}

The propagation matrix will usually contain a contribution from the background continuum opacity which we assume here to be unpolarized. The propagation matrix is then of the form

$\boldsymbol{K}=\kappa_{\mathrm{c}} \boldsymbol{E}+\kappa_{\mathrm{o}} \boldsymbol{\Phi}$

where $\kappa_{\mathrm{c}}$ is the continuum opacity, assumed to be independent of frequency, $\kappa_{\mathrm{O}}$ the frequency integrated line opacity and $\boldsymbol{\Phi}$ the spectral line propagation matrix. We assume that the continuum and line source functions are identical and given by the Planck function. We introduce the ratio $\kappa_{\mathrm{o}} / \kappa_{\mathrm{c}}=\beta$, with $\beta$ a constant, and the continuum optical depth $\mathrm{d} \tau_{\mathrm{c}}=\kappa_{\mathrm{c}} \mathrm{d} s$ which is now used as the space variable. The radiative transfer equation can then be written as

$\frac{\mathrm{d} \boldsymbol{I}}{\mathrm{d} \tau_{\mathrm{c}}}=[\boldsymbol{E}+\beta \boldsymbol{\Phi}][\boldsymbol{I}-\boldsymbol{S}]$.

We assume that the Planck function is linear in $\tau_{\mathrm{c}}$ and write $\boldsymbol{S}\left(\tau_{\mathrm{c}}\right)=\left(B_{0}+B_{1} \tau_{\mathrm{c}}\right) \boldsymbol{U}$ with $\boldsymbol{U}=[1,0,0,0]^{\mathrm{T}}$. The assumptions of a constant $\beta$ and a linear source function are characteristic of a Milne-Eddington model.

At the surface, the Stokes vector in the continuum is given by

$\boldsymbol{I}_{\mathrm{c}}(0)=\left(B_{0}+B_{1}\right) \boldsymbol{U}$.

With our choice for $\boldsymbol{U}$, only the first component of $\boldsymbol{I}_{\mathrm{c}}$, i.e. the intensity component $I_{\mathrm{c}}$, is non zero.

Equation (16) shows that the magnetic field effects are contained in $\langle\tilde{\boldsymbol{O}}(0,0)\rangle_{\mathrm{KA}}$. This suggests to introduce

$\boldsymbol{r}(0) \equiv[\boldsymbol{E}-\tilde{\boldsymbol{O}}(0,0)] \boldsymbol{U}=\frac{1}{B_{1}}\left[\boldsymbol{I}_{\mathrm{c}}(0)-\boldsymbol{I}(0)\right]$

with $\tilde{\boldsymbol{O}}(0,0)$ the Laplace transform for $p=0$ of the propagation operator (see Eq. (11)). For simplicity, $\boldsymbol{r}(0)$ will be referred to as the residual Stokes vector, although the usual residual Stokes vector, also called line depression Stokes vector (Stenflo 1994$, p. 244$)$, is defined as $\left[\boldsymbol{I}_{\mathrm{c}}(0)-\boldsymbol{I}(0)\right] / I_{\mathrm{c}}(0)$. Equations (16) and (17) yield

$$
\begin{aligned}
\langle\boldsymbol{r}(0)\rangle_{\mathrm{KA}} & =\left[\boldsymbol{E}-\left\langle\tilde{\boldsymbol{O}}_{\mathrm{S}}(v)\right\rangle\left[\boldsymbol{E}-v\left\langle\tilde{\boldsymbol{O}}_{\mathrm{S}}(v)\right\rangle\right]^{-1}\right] \boldsymbol{U} \\
& =\left[\boldsymbol{E}-(1+v)\left\langle\tilde{\boldsymbol{O}}_{\mathrm{S}}(v)\right\rangle\right]\left[\boldsymbol{E}-v\left\langle\tilde{\boldsymbol{O}}_{\mathrm{S}}(v)\right\rangle\right]^{-1} \boldsymbol{U},
\end{aligned}
$$

where

$\left\langle\tilde{\boldsymbol{O}}_{\mathbf{S}}(v)\right\rangle=\frac{1}{1+v}\left\langle\left[\boldsymbol{E}+\frac{\beta}{1+v} \boldsymbol{\Phi}\right]^{-1}\right\rangle$. 
The expression of $\left\langle\tilde{\boldsymbol{O}}_{\mathrm{S}}(v)\right\rangle$ follows from Eq. (13) where we have set $p=v$ and $\boldsymbol{K}=\boldsymbol{E}+\beta \boldsymbol{\Phi}$.

The mean residual Stokes vector can also be written as

$$
\begin{aligned}
& \langle\boldsymbol{r}(0)\rangle_{\mathrm{KA}}=(1+v) \\
& \boldsymbol{R}_{\text {macro }}\left(\frac{\beta}{1+v} \boldsymbol{\Phi}\right)\left[\boldsymbol{E}+v \boldsymbol{R}_{\text {macro }}\left(\frac{\beta}{1+v} \boldsymbol{\Phi}\right)\right]^{-1} \boldsymbol{U},
\end{aligned}
$$

where

$\boldsymbol{R}_{\text {macro }}(\lambda \boldsymbol{\Phi}) \equiv\left\langle\lambda \boldsymbol{\Phi}[\boldsymbol{E}+\lambda \boldsymbol{\Phi}]^{-1}\right\rangle$,

with $\lambda$ a scalar.

In the macroturbulent and microturbulent limits, Eq. (29) reduces to

$\langle\boldsymbol{r}(0)\rangle_{\text {macro }}=\boldsymbol{R}_{\text {macro }}(\beta \boldsymbol{\Phi}) \boldsymbol{U}$,

$$
\langle\boldsymbol{r}(0)\rangle_{\text {micro }}=\beta\langle\boldsymbol{\Phi}\rangle[\boldsymbol{E}+\beta\langle\boldsymbol{\Phi}\rangle]^{-1} \boldsymbol{U} .
$$

The microturbulent limit is readily obtained by subtracting $\boldsymbol{I}_{\mathrm{c}}(0)$ from Eq. (22). The mean value $\langle\boldsymbol{\Phi}\rangle$ has been investigated in some detail for random magnetic fields with isotropic and anisotropic Gaussian fluctuations in Frisch et al. (2005, henceforth Paper I) (see also Dolginov \& Pavlov 1972; Domke \& Pavlov 1979; Frisch et al. 2006; Sampoorna et al. 2006).

The expressions given here for the residual Stokes vector are similar to the expressions given in Auvergne et al. (1973) for the broadening by a turbulent velocity field. The only difference is that the line absorption coefficient is now a matrix instead of a simple scalar. From a numerical point of view, it is more convenient to work with the residual Stokes vector than with the Stokes vector itself because the averaging is done on quantities which go to zero at large frequencies.

\section{Remark}

In the proof given above we have assumed for simplicity that randomness in $\boldsymbol{\Phi}$, and thus in $\boldsymbol{K}$, comes only from the magnetic field. If randomness comes from other physical parameters and provided they are described with the same type of random process as the magnetic field, in particular the same correaltion length, all the theoretical results given here will still hold, but the averaging over $P(\boldsymbol{H})$ must be replaced by an averaging over a joint probability distribution function $P\left(\boldsymbol{H}, \alpha_{1}, \alpha_{2}, \ldots\right)$, where the $\alpha_{i}$ are scalar or vector random parameters. This remark holds also for the results in Sect. 4 on the second-order moments.

\section{Numerical evaluation of the mean Stokes parameters}

In this section we use Eq. (29) to study the dependence of $\langle\boldsymbol{r}(0)\rangle_{\mathrm{KA}}$ on the correlation length of a random magnetic field with isotropic Gaussian fluctuations. We assume that the velocity field is microturbulent. Its effects can thus be incorporated in the definition of the profile $\boldsymbol{\Phi}$ and of the Doppler width. This assumption allows us to clearly identify the effects of the random magnetic field. The function $P(\boldsymbol{H})$ is defined in Sect. 3.1 and numerical results are presented in Sect. 3.2.

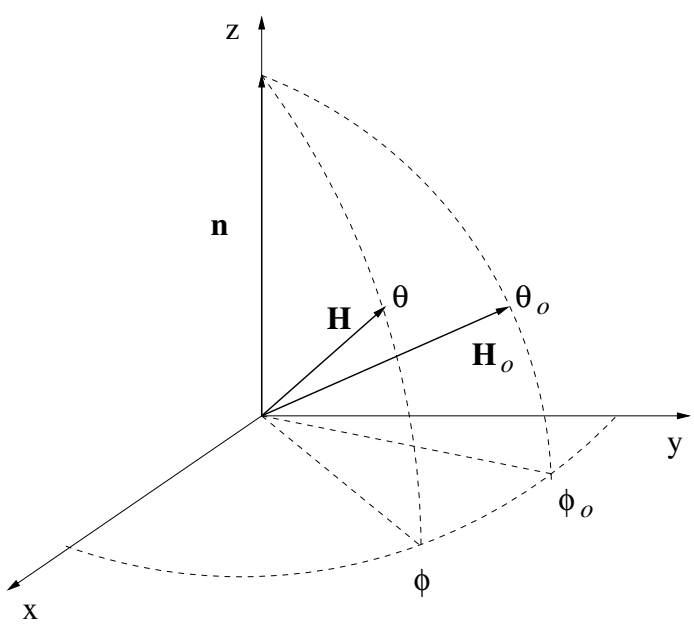

Fig. 1. Definition of $\theta, \phi, \theta_{0}$ and $\phi_{0}$, the inclinations and longitudes of the random magnetic field vector $\boldsymbol{H}$, and of its mean $\boldsymbol{H}_{0}$. The angles $\theta$ and $\theta_{0}$ are defined with respect to the direction $z$ along the line of sight.

\subsection{Probability distribution function of the vector magnetic field}

To calculate $\langle\boldsymbol{r}(0)\rangle_{\mathrm{KA}}$ we must perform the averaging over $P(\boldsymbol{H})$ of the r.h.s. in Eq. (30) where $\lambda=\beta /(1+v)$. For a random magnetic field with isotropic Gaussian fluctuations,

$$
\begin{aligned}
& P(\boldsymbol{H}) \mathrm{d} \boldsymbol{H}= \\
& \frac{1}{(2 \pi)^{3 / 2} \sigma^{3}} \exp \left[\frac{-\left(\boldsymbol{H}-\boldsymbol{H}_{0}\right)^{2}}{2 \sigma^{2}}\right] H^{2} \sin \theta \mathrm{d} \theta \mathrm{d} \phi \mathrm{d} H,
\end{aligned}
$$

where $\boldsymbol{H}_{0}$ is the mean value of $\boldsymbol{H}$ and $3 \sigma^{2}=\left\langle\left(\boldsymbol{H}-\boldsymbol{H}_{0}\right)^{2}\right\rangle$, the dispersion around the mean value. The angles $\theta$ and $\phi$ are the inclination and longitude of the random magnetic field with respect to the line of sight (see Fig. 1). The direction of the mean field is defined by the angles $\theta_{0}$ and $\phi_{0}$. The amplitudes of $\boldsymbol{H}$ and $\boldsymbol{H}_{0}$ are denoted by $H$ and $H_{0}$. The Zeeman shift by the mean magnetic field is $\Delta H_{0}$. Here $\Delta=g \frac{e}{4 \pi m c} \frac{1}{\Delta_{\mathrm{D}}}$, with $g$ the Landé factor, $e$ and $m$ the mass and charge of the electron, $c$ the speed of light and $\Delta_{\mathrm{D}}$ the Doppler width. Frequencies, denoted by $x$, are measured in units of the Doppler width, with zero at line center. The Doppler width is given by $\Delta_{\mathrm{D}}=v_{0}\left(v_{\text {th }}^{2}+v_{\text {turb }}^{2}\right)^{1 / 2} / c$, where $v_{\text {turb }}$ and $v_{\text {th }}$ are the rms microturbulent and thermal velocity and $v_{0}$ is the frequency at line center. The usual factor $1 / \sqrt{\pi}$ is absorbed in the definition of $\boldsymbol{\Phi}$ (see Paper I).

The effects of the random magnetic field are controlled by two parameters:

$y_{0} \equiv \frac{H_{0}}{\sqrt{2} \sigma} \quad$ and $\quad \gamma_{\mathrm{H}} \equiv \Delta \sqrt{2} \sigma \quad$ or $\quad \Delta H_{0}$

where $\Delta H_{0}$ is the Zeeman shift by the mean magnetic field and $\gamma_{\mathrm{H}}$ the Zeeman shift by the rms fluctuations, also measured in Doppler width unit, which acts as a magnetic broadening on the $\sigma$-components of the Zeeman propagation matrix. The parameter $y_{0}=H_{0} / \sqrt{2} \sigma=\Delta H_{0} / \gamma_{\mathrm{H}}$, is the ratio of these two shifts. When $y_{0}$ is smaller than unity $\left(y_{0}<0.1-0.2\right), \Delta H_{0}$ is smaller than the combined Doppler and random magnetic field broadening measured by the parameter $\gamma_{1}=\sqrt{1+\gamma_{\mathrm{H}}^{2}}$. In this limit, the $\sigma$-components are not resolved. In contrast, when $y_{0}$ is larger than unity, say $y_{0}>2$, the $\sigma$-components are well separated, provided $\Delta H_{0}$ is larger than unity. We also introduce $f=1 / y_{0}$ which measures the strength of the turbulent fluctuations, large 


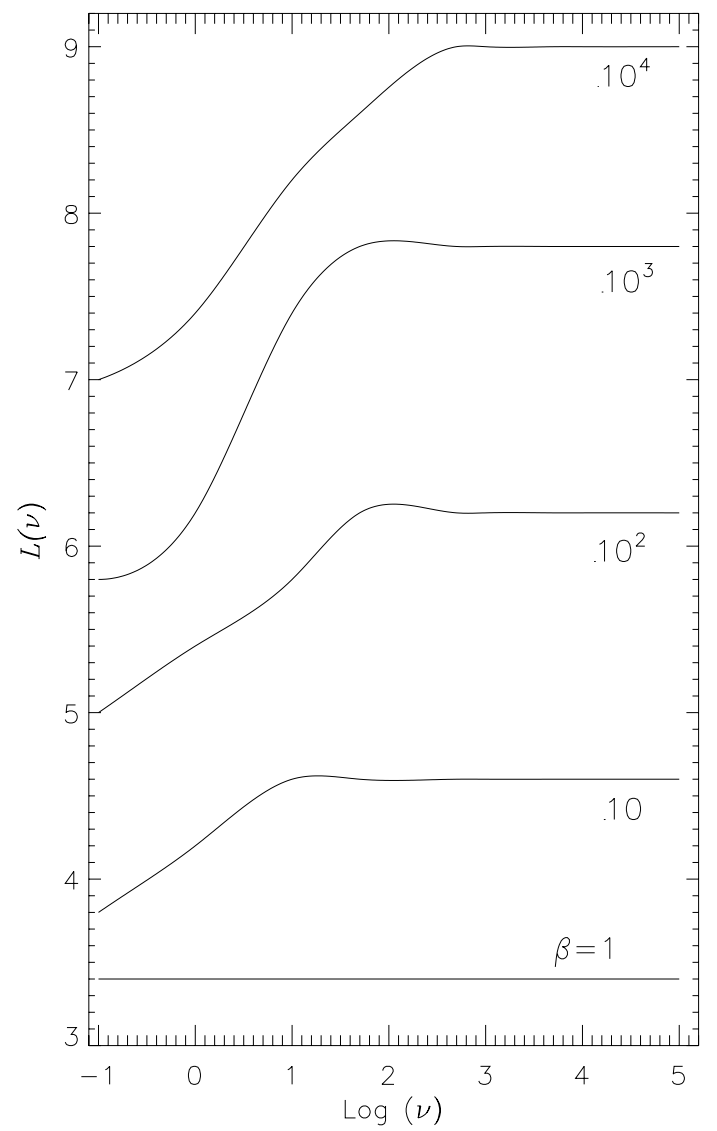

Fig. 2. Variation of the full width at half maximum $L(v)$ of the emergent Stokes $I$ profile with the jump frequency $v$ for various values of the line strength $\beta$. The model parameters employed are $y_{0}=\Delta H_{0}=0.1$; $\gamma_{\mathrm{H}}=1 ; \theta_{0}=0^{\circ} ; \phi_{0}=0^{\circ}$ and $a=0$.

values corresponding to strong turbulence and small ones to weak turbulence.

\subsection{Numerical results. Effects of a finite correlation length}

The numerical method for averaging over $P(\boldsymbol{H})$ is described in Paper I, where it is applied to the calculation of $\langle\boldsymbol{\Phi}\rangle$. Although the expressions here are somewhat more complicated, the same technique can be applied. The averaging involves a triple integration over the variables $y=H / \sqrt{2} \sigma, \theta$ and $\phi$. The $y$-integration requires some care. It is performed using a Gauss-Legendre quadrature formula with 10 to 30 points in a range $\left[0,2 y_{\max }\right]$. We have choosen $y_{\max }=1$ for $y_{0}<1$ and $y_{\max }=y_{0}$ for $y_{0}>1$. The mean residual Stokes parameters are calculated in a frequencybandwidth $\left[-x_{\max },+x_{\max }\right]$ with $x_{\max }=4 \gamma_{\mathrm{H}} y_{\max }$. All the calculations reported here are performed with a damping parameter $a=0$. In Paper I it is shown that the elements of $\langle\boldsymbol{\Phi}\rangle$ are not very sensitive to the value of $a$, unless it becomes larger than 0.1 .

Equation (29) shows that $\langle\boldsymbol{r}(0)\rangle_{\mathrm{KA}}$ involves the parameter $v$ and the ratio $\beta /(1+v)$. When $\beta$ is small, and a fortiori $\beta /(1+v)$, Eq. (28) (or (30)) shows that $\frac{\beta}{1+v} \boldsymbol{\Phi}$ can be neglected compared to the identity matrix. Hence, for small values of $\beta,\langle\boldsymbol{r}\rangle_{\text {micro }} \simeq$ $\langle\boldsymbol{r}\rangle_{\text {macro }} \simeq \beta\langle\boldsymbol{\Phi}\rangle \boldsymbol{U}$. Therefore for weak lines, the Stokes parameters depend only on $P(\boldsymbol{H})$ (through $\langle\boldsymbol{\Phi}\rangle$ ) in the region of the line formation. For lines sensitive to the value of $v$, the microturbulent regime is reached when $\beta / v<1$, i.e. when the correlation length has a line optical depth smaller than unity. These remarks are illustrated in Fig. 2 which shows $L(v)$, the full frequency
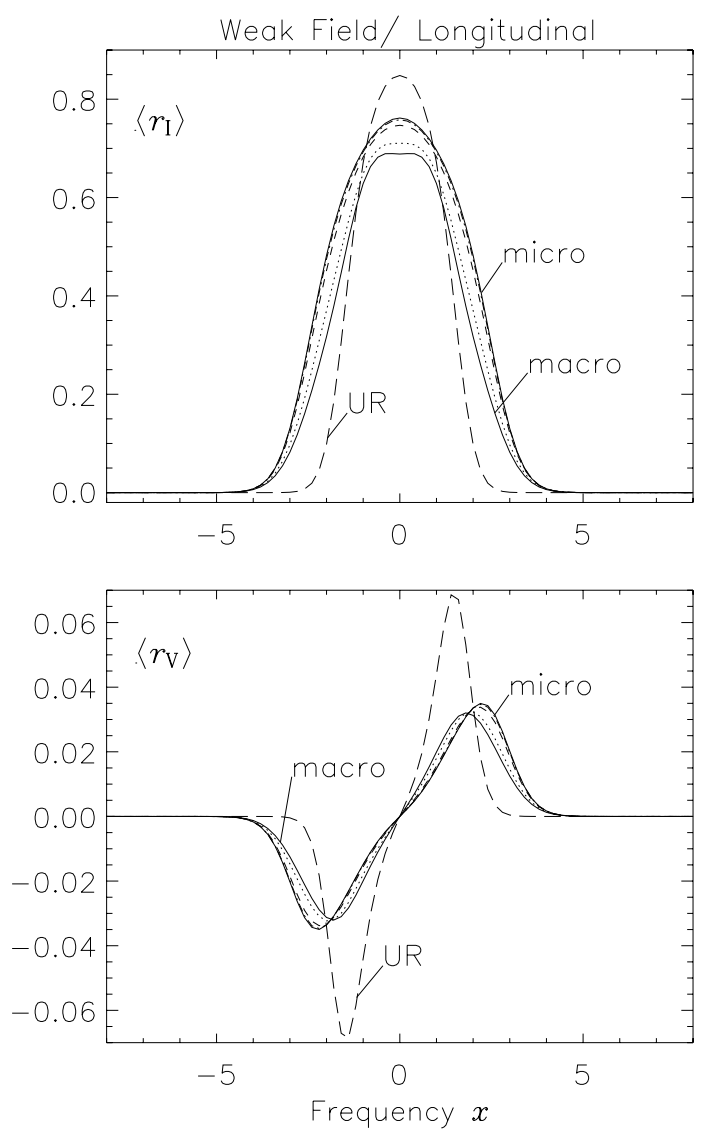

Fig. 3. Dependence of the mean Stokes parameters on the correlation length $1 / v$ for a weak mean magnetic field and strong turbulence $(f=10)$. The model parameters are: $\beta=10, \Delta H_{0}=0.1, \gamma_{\mathrm{H}}=1$. The mean field $\boldsymbol{H}_{0}$ is in the direction of LOS. The full lines show the macro $(v=0)$ and micro limits. The line types are: dotted $(v=1)$; dashed $(v=10)$; dot-dashed $(v=50)$. The long-dashed lines correspond to the Unno-Rachkovsky (UR) solution calculated with $\boldsymbol{H}_{0}$.

width at half-maximum of $\langle I(0)\rangle_{\mathrm{KA}}$ (the mean value of Stokes $I$ ), for different choices of $\beta$ and $v$. We have assumed $\Delta H_{0}=0.1$ and $\gamma_{\mathrm{H}}=1$, in order to have for Stokes $I$ a single well defined peak allowing for an unambiguous definition of $L(v)$. Figure 2 clearly shows that the dependence on $v$ increases with $\beta$ and that the microturbulent regime, indicated by the fact that $L(v)$ reaches a constant value, sets in at roughly $v \simeq \beta$.

Numerical results illustrating the $v$ dependence of $\left\langle r_{I}\right\rangle,\left\langle r_{V}\right\rangle$ and $\left\langle r_{Q}\right\rangle$, the Stokes $I, Q$ and $V$ components of $\langle\boldsymbol{r}(0)\rangle_{\mathrm{KA}}$, are shown in Figs. 3 to 9 for different values of $\beta$ (10 and 100) and different magnetic field parameters. To simplify the notation, without risk of confusion, we have omitted the subscript "KA" and the value $\tau_{\mathrm{c}}=0$ for the components of $\langle\boldsymbol{r}(0)\rangle_{\mathrm{KA}}$. In all the figures $\gamma_{\mathrm{H}}=\Delta \sqrt{2} \sigma=1$, which means that the random magnetic field broadening is of the same order as the broadening by the combined thermal and turbulent velocities. Hence the strength of the fluctuations is always $f=1 / \Delta H_{0}$. For comparison we also show the Unno-Rachkovsky solution calculated with the mean field $\boldsymbol{H}_{0}$, henceforth referred to as the mean Unno-Rachkovsky solution and denoted UR. The relative variation between the micro and macroturbulent limits are evaluated by considering the ratio $\delta\left\langle r_{X}\right\rangle=\left[\left|\left\langle r_{X}\right\rangle_{\text {micro }}-\left\langle r_{X}\right\rangle_{\text {macro }}\right|\right] /\left|\left\langle r_{X}\right\rangle_{\text {micro }}\right|$ where the subscript $X$ stands for $I, Q$ or $V$.

(i) Behavior of $\left\langle r_{\mathrm{I}}\right\rangle$. All Figs. 3 to 9 clearly show that the profiles corresponding to a finite value of $v$ lie, as expected, between the microturbulent and macroturbulent limits, with the 

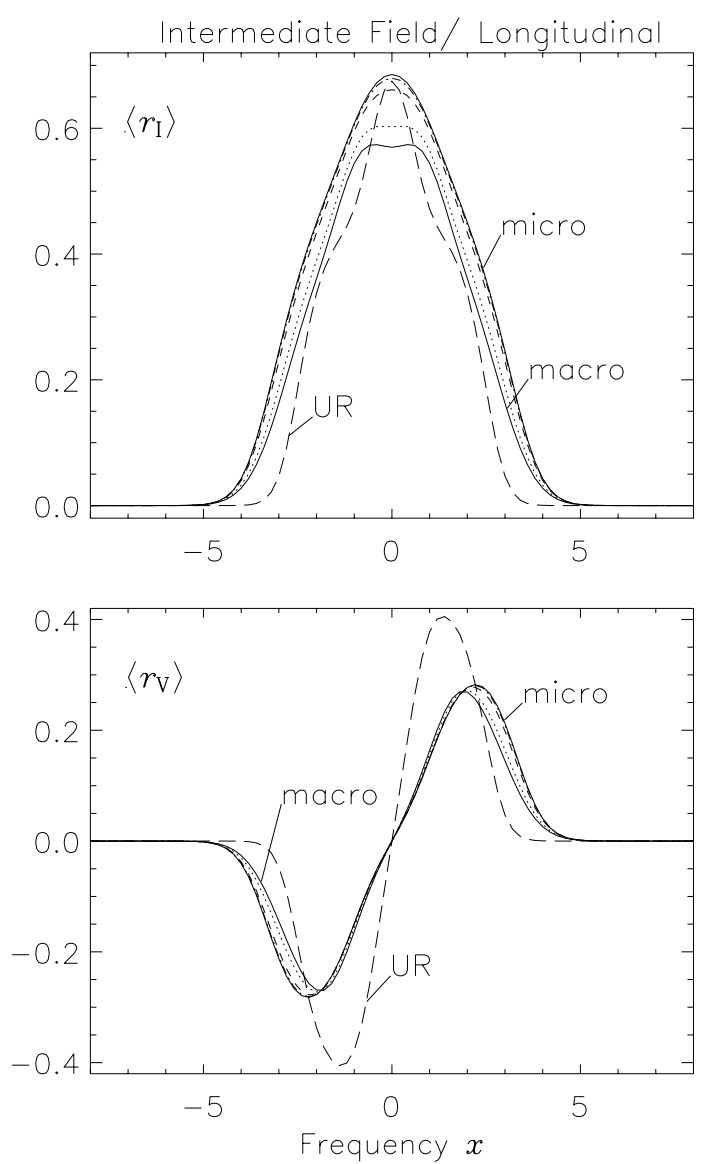

Fig. 4. Same as Fig. 3 but for moderate turbulence $(f=1)$. The model parameters are: $\beta=10, \Delta H_{0}=1, \gamma_{\mathrm{H}}=1$.

microturbulent profiles being at all frequencies broader than the macroturbulent ones, especially around the frequencies corresponding to the $\sigma$-components. When $\beta=10$ (Figs. 3 to 7), the relative variations, measured with $\delta\left\langle r_{I}\right\rangle$, are between $10 \%$ and $20 \%$ at line center and also in the $\sigma$-components, when the latter are well separated. The main trend at line center is an increase of $\delta\left\langle r_{I}\right\rangle$ with $\Delta H_{0}$. The value of $f$ seems to be essentially irrelevant. In Fig. 6, where the mean field is longitudinal, $\left\langle r_{I}\right\rangle$ shows an unpolarized $\pi$-component created by the angular averaging of the $\sin ^{2} \theta$ factor in the $\pi$-component of the absorption coefficient (see Paper I). The strength of this component is very sensitive to the angular distribution of the magnetic field fluctuations.

When $\beta=100$ (Figs. 8 and 9), $\left\langle r_{I}\right\rangle$ deviates strongly from the UR solution. When $\boldsymbol{H}_{0}$ is longitudinal (Fig. 8), a peak appears at line center and its value is almost independent of the correlation length. As shown by the Unno-Rachkovsky solution the central component behaves essentially as $\beta\left\langle\varphi_{\mathrm{I}}\right\rangle /\left(1+\beta\left\langle\varphi_{\mathrm{I}}\right\rangle\right)$, with $\left\langle\varphi_{\mathrm{I}}\right\rangle$ the mean value of the absorption coefficient. At line center, when the magnetic field is random, $\left\langle\varphi_{\mathrm{I}}\right\rangle$ becomes much larger than its deterministic counterpart calculated with $\boldsymbol{H}_{0}$. Hence when $\beta$ is fairly large, the value of the central peak may approach unity. When $\boldsymbol{H}_{0}$ is in the transverse direction, one observes drastic changes between the macroturbulent and microturbulent limits which can also be explained in terms of the behavior of $\left\langle\varphi_{\mathrm{I}}\right\rangle$.

(ii) Behavior of $\left\langle r_{V}\right\rangle$. A striking feature (see Figs. 3, 4, $6,8)$ is the strong deviation from the UR solution for strong and moderate turbulence (see Figs. 3 and 4 with $f=10$ and $f=1$ ) while for weak turbulence, $\left\langle r_{V}\right\rangle$ stays very close to the
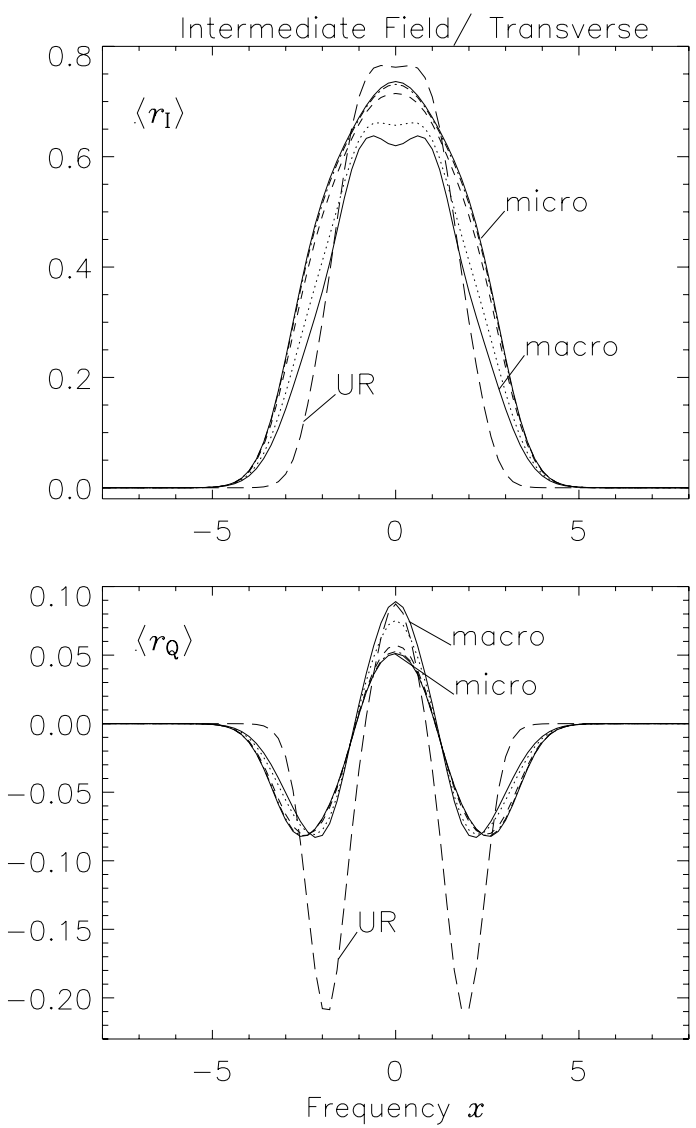

Fig. 5. Same as Fig. 4, but with the mean field $\boldsymbol{H}_{0}$ perpendicular to the direction of the LOS.

UR solution (see Figs. 6 and 8 with $f=1 / 3$ ). The relative variations between the micro and macroturbulent limits seems to be largely independent of the value of $f$. They are always smaller than $10 \%$ and in general smaller than the variation of $\left\langle r_{I}\right\rangle$ at line center, except for the case of Fig. 3 where they are both of the same order and slightly less than $10 \%$. It thus seems that $\left\langle r_{V}\right\rangle$, can be calculated with the microturbulent limit, with reasonable confidence, ignoring the correlation length of the magnetic field.

(iii) Behavior of $\left\langle r_{Q}\right\rangle$. Figures 5, 7, 9 show a strong deviation from the UR solution which decreases when the strength of the turbulent fluctuations decreases. For $\beta=10$, at line center $\delta\left\langle r_{Q}\right\rangle$ reaches $75 \%$ when $f=1$ but decreases to $20 \%$ when $f=1 / 3$. For this value of $\beta$, one can observe that the line center is more sensitive to the correlation length than the $\sigma$-components. For $\beta=100$ and although $f=1 / 3$ only (see Fig. 9), $\left\langle r_{Q}\right\rangle$ is very sensitive to the correlation length, at line center and also in the wings. At line center, $\left\langle r_{Q}\right\rangle$ is bounded by the macro and microturbulent limits, but in the $\sigma$-components the behavior is not so simple because the position of the peaks moves away from the line center when $v$ increases. The maximum depth of the $\sigma$-components stays however always above the macroturbulent value. Finally we remark that for weak fluctuations $(f=1 / 3)$, $\left\langle r_{Q}\right\rangle$ will depart more from the UR solution than $\left\langle r_{V}\right\rangle$ (compare Figs. 6 and 7).

All the figures shown in this section confirm the remark that microturbulence is reached when $\beta / v \simeq 1$. 

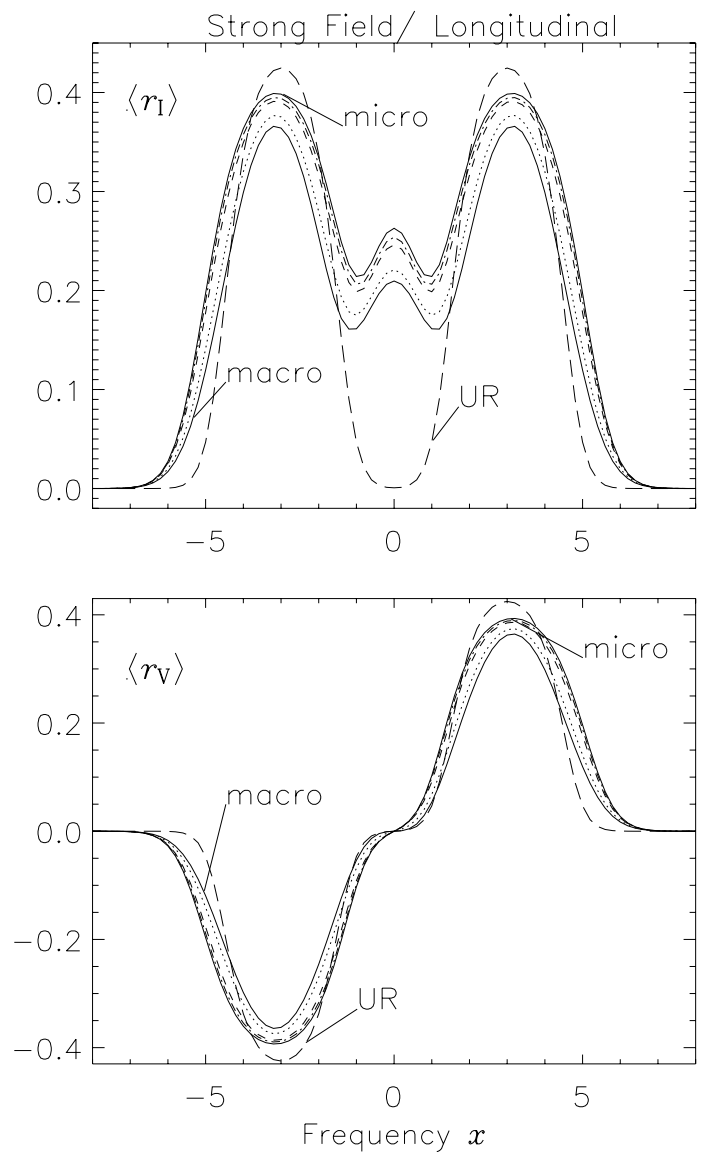

Fig. 6. Dependence of the mean Stokes parameters on the correlation length $1 / v$ of the magnetic field for a strong mean field and weak turbulence $(f=1 / 3)$. The model parameters are: $\beta=10, \Delta H_{0}=3, \gamma_{\mathrm{H}}=1$. The mean field $\boldsymbol{H}_{0}$ is in the direction of the LOS. The line types have the same meaning as in Fig. 3.

\section{Second-oder moments and dispersion of the Stokes parameters}

We now examine the fluctuations of the Stokes parameters around their mean values. For each Stokes parameter, we consider the square of the dispersion,

$\sigma_{i}^{2}(0) \equiv\left\langle I_{i}^{2}(0)\right\rangle_{\mathrm{KA}}-\left\langle I_{i}(0)\right\rangle_{\mathrm{KA}}^{2}$,

where $I_{i}$ stands for $I, Q, U$ or $V$. To calculate these quantities, we must consider second-order moments of the Stokes parameters, i.e. quantities of the form $\left\langle I_{i}(0) I_{j}(0)\right\rangle_{\mathrm{KA}}$.

Second-order moments are investigated in Brissaud \& Frisch (1974) for systems of linear stochastic equations, but only for homogeneous systems or systems with a white noise inhomogeneous term. Here we show that explicit expressions for secondorder moments can also be obtained for inhomogeneous systems with a constant inhomogeneous term. Our method is inspired by Brissaud \& Frisch (1974).

When the source function $S\left(\tau_{\mathrm{c}}\right)$ varies linearly with optical depth, one can easily obtain a vector transfer equation with a constant inhomogeneous term. It suffices to introduce the new unknown vector

$\boldsymbol{Y}\left(\tau_{\mathrm{c}}\right) \equiv \boldsymbol{I}\left(\tau_{\mathrm{c}}\right)-\boldsymbol{S}\left(\tau_{\mathrm{c}}\right)$
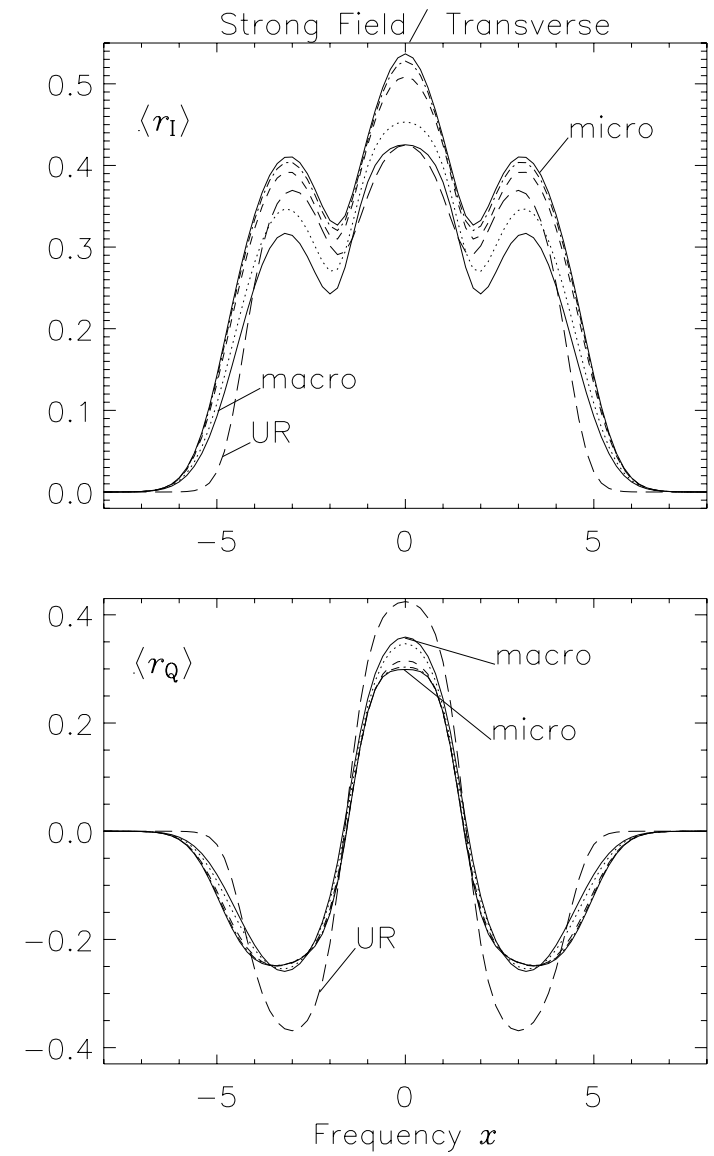

Fig. 7. Same as Fig. 6, but with the mean field $\boldsymbol{H}_{0}$ perpendicular to the direction of the LOS.

Since $\boldsymbol{S}$ is non-random, $\boldsymbol{I}$ and $\boldsymbol{Y}$ will have the same dispersion. The vector $\boldsymbol{Y}$ satisfies the transfer equation

$\frac{\mathrm{d} \boldsymbol{Y}}{\mathrm{d} \tau_{\mathrm{c}}}=[\boldsymbol{E}+\beta \boldsymbol{\Phi}] \boldsymbol{Y}-B_{1} \boldsymbol{U}=\boldsymbol{K} \boldsymbol{Y}-B_{1} \boldsymbol{U}$,

where the inhomogeneous term $B_{1} \boldsymbol{U}$ is a constant vector. In this section, to simplify the notation, we set $\tau_{\mathrm{c}}=s$. The solution of Eq. (37) can be written as

$\boldsymbol{Y}(s)=B_{1}\left[\int_{s}^{\infty} \boldsymbol{O}\left(s, s^{\prime}\right) \mathrm{d} s^{\prime}\right] \boldsymbol{U}$,

where $\boldsymbol{O}\left(s, s^{\prime}\right)$ has been introduced in Sect. 2 as the propagation operator for Eq. (1).

In Sect. 4.1, we use Eq. (38) to establish a transfer equation for the tensor product $\boldsymbol{Y}(s) \otimes \boldsymbol{Y}(s)$ and solve it for $\boldsymbol{Y}(0) \otimes \boldsymbol{Y}(0)$. In Sect. 4.2 we establish an explicit expression for $\langle\boldsymbol{Y}(0) \otimes \boldsymbol{Y}(0)\rangle_{\mathrm{KA}}$ by a summation method and use it in Sect. 4.3 to illustrate the dependence of the dispersion on the correlation length and strength of the magnetic field fluctuations.

\subsection{Transfer equation for the second-order moment of the Stokes vector}

To calculate the dispersions $\sigma_{i}^{2}(0)$, we need only $\left\langle Y_{i}(0) Y_{i}(0)\right\rangle_{\mathrm{KA}}$, however the latter cannot be calculated independently of the other $\left\langle Y_{i}(0) Y_{j}(0)\right\rangle_{\mathrm{KA}}$. We therefore introduce the tensor product

$\boldsymbol{Y}(s) \otimes \boldsymbol{Y}(s)=Y_{i}(s) Y_{j}(s), \quad$ with $\quad i, j=1,4$. 

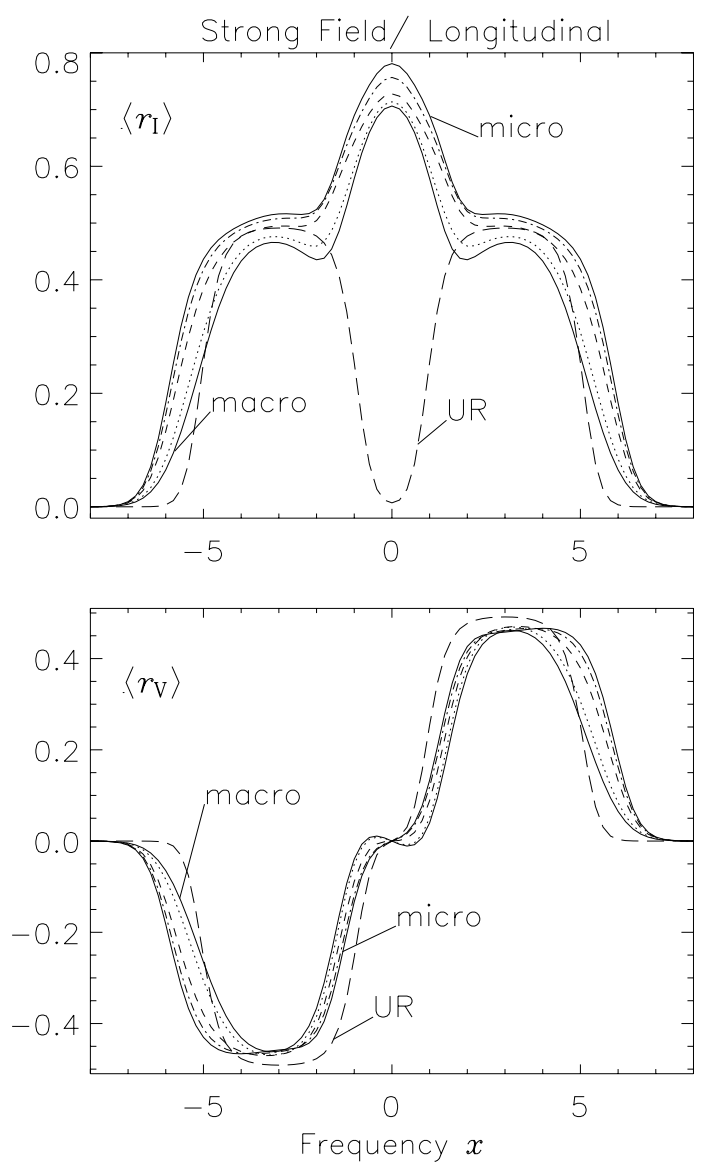

Fig. 8. Dependence of the mean Stokes parameters on the correlation length $1 / v$ of the magnetic field for a strong line: $\beta=100$. The other model parameters are $\Delta H_{0}=3$ and $\gamma_{\mathrm{H}}=1$. They are the same as in Fig. 6 and correspond to a weak turbulence case $(f=1 / 3)$. The mean field $\boldsymbol{H}_{0}$ is in the direction of the LOS. The line types have the same meaning as in Fig. 3.

We associate the indices 1 to 4 to $I, Q, U$ and $V$, respectively. We consider $Y_{i}(s) Y_{j}(s)$ to be the components of a 16-dimension vector. For symmetry reasons, there is actually only 10 different components. One could also consider $Y_{i}(s) Y_{j}(s)$ to be elements of a $4 \times 4$ matrix. However there is no real advantage to work with a matrix and furthermore such a description will not hold for third or higher moments.

It follows from Eq. (37) that $\boldsymbol{Y}(s) \otimes \boldsymbol{Y}(s)$ satisfies the transfer equation

$$
\begin{aligned}
& \frac{\mathrm{d}}{\mathrm{d} s}[\boldsymbol{Y}(s) \otimes \boldsymbol{Y}(s)]= \\
& \quad \boldsymbol{K} \boldsymbol{Y} \otimes \boldsymbol{Y}+\boldsymbol{Y} \otimes \boldsymbol{K} \boldsymbol{Y}-B_{1}(\boldsymbol{U} \otimes \boldsymbol{Y}+\boldsymbol{Y} \otimes \boldsymbol{U}) .
\end{aligned}
$$

We recall that the tensor product, also called Kronecker product (Iyanaga $\&$ Kawada 1970, p. 851), of a $m \times n$ matrix $C$ by a $r \times s$ matrix $D$ is a $m r \times n s$ matrix which can be written as

$C \otimes D=\left[\begin{array}{ccc}C_{11} D & \ldots & C_{1 n} D \\ \vdots & \ddots & \vdots \\ C_{m 1} D & \ldots & C_{m n} D\end{array}\right]$.

A useful formula satisfied by tensor products is

$\left(C_{1} \otimes D_{1}\right)\left(C_{2} \otimes D_{2}\right)=C_{1} C_{2} \otimes D_{1} D_{2}$,
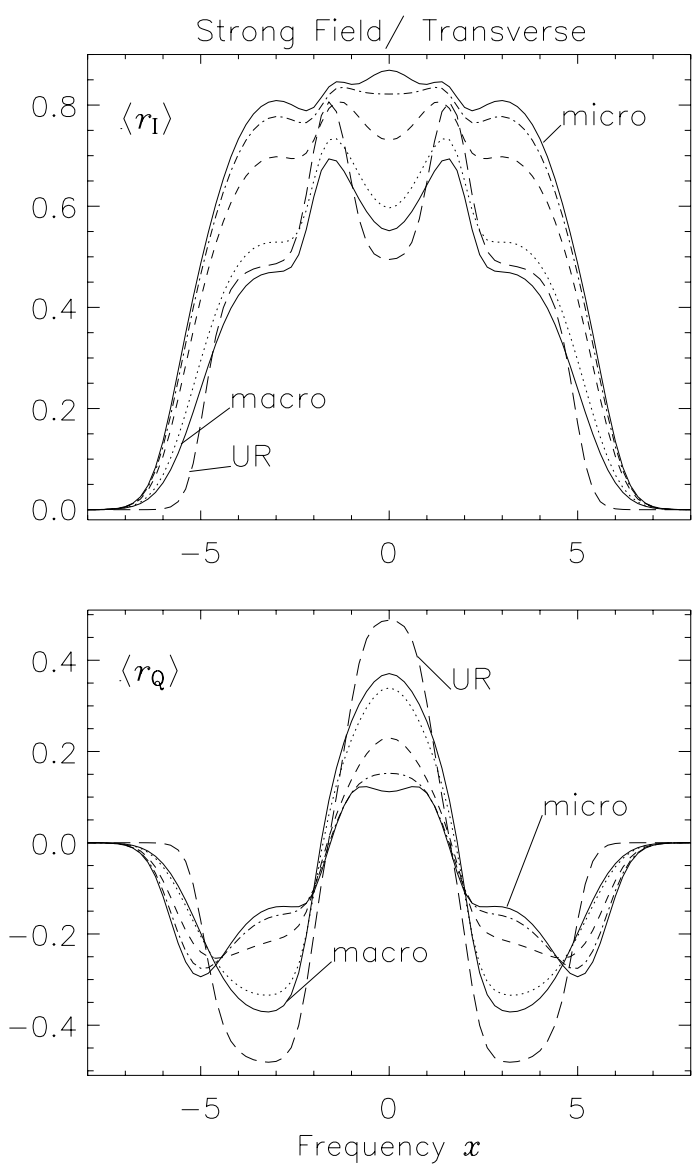

Fig. 9. Same as Fig. 8, but with the mean field $\boldsymbol{H}_{0}$ perpendicular to the direction of the LOS.

provided the matrix products can be defined. It is used here several times with one of the matrix, say $C_{1}$, equal to the identity matrix $E$. In that case,

$\left(E \otimes D_{1}\right)\left(C_{2} \otimes D_{2}\right)=C_{2} \otimes D_{1} D_{2}$.

It follows from Eq. (43), that Eq. (40) can be rewritten as

$\frac{\mathrm{d}}{\mathrm{d} s}[\boldsymbol{Y}(s) \otimes \boldsymbol{Y}(s)]=\mathcal{K}(\boldsymbol{Y} \otimes \boldsymbol{Y})-B_{1} \boldsymbol{Y}$,

where

$\boldsymbol{Y} \equiv \boldsymbol{U} \otimes \boldsymbol{Y}+\boldsymbol{Y} \otimes \boldsymbol{U}$

$\mathcal{K} \equiv \boldsymbol{K} \otimes \boldsymbol{E}+\boldsymbol{E} \otimes \boldsymbol{K}$

with $\mathcal{Y}$ a 16 -dimension vector and $\mathcal{K}$ a $16 \times 16$ matrix. We use calligraphic letters to denote $16 \times 16$ matrices and 16 -dimension vectors (the indices run from 1 to 16 ).

The Green's function (or propagation operator) $\mathcal{G}\left(s, s^{\prime}\right)$ associated to Eq. (44) satisfies

$\frac{\mathrm{d}}{\mathrm{d} s} \mathcal{G}\left(s, s^{\prime}\right)=\mathcal{K}(s) \mathcal{G}\left(s, s^{\prime}\right), \quad$ with $\quad \mathcal{G}(s, s)=\mathcal{E}$,

where $\mathcal{E}$ is the $16 \times 16$ identity matrix. The function $\mathcal{G}\left(s, s^{\prime}\right)$ has a static version $\mathcal{G}_{\mathrm{S}}(s)$ corresponding to $\mathcal{K}$ (i.e. $\boldsymbol{K}$ ) independent of $s$. Combining the transfer equation for $\boldsymbol{O}\left(s, s^{\prime}\right)$ (identical to Eq. (47) with $\mathcal{K}(s)$ replaced by $\boldsymbol{K}(s)$ ), Eqs. (46) and (47), one can show that

$\mathcal{G}\left(s, s^{\prime}\right)=\boldsymbol{O}\left(s, s^{\prime}\right) \otimes \boldsymbol{O}\left(s, s^{\prime}\right)$. 
We note that in Brissaud \& Frisch (1974), $\mathcal{G}\left(s, s^{\prime}\right)$ is referred to as the double Green's function.

In terms of $\mathcal{G}\left(s, s^{\prime}\right)$, the solution of Eq. (44) at the surface may be written as

$\boldsymbol{Y}(0) \otimes \boldsymbol{Y}(0)=B_{1} \int_{0}^{\infty} \mathcal{G}(0, s) \boldsymbol{Y}(s) \mathrm{d} s$.

Using now Eqs. (38), (43) and (45), we obtain

$$
\begin{aligned}
& \boldsymbol{Y}(0) \otimes \boldsymbol{Y}(0)=B_{1}^{2}\left[\int_{0}^{\infty} \mathcal{G}(0, s)\right. \\
& \left.\times \int_{s}^{\infty}\left[\boldsymbol{O}^{\mathrm{l}}\left(s, s^{\prime}\right)+\boldsymbol{O}^{\mathrm{r}}\left(s, s^{\prime}\right)\right] \mathrm{d} s^{\prime} \mathrm{d} s\right](\boldsymbol{U} \otimes \boldsymbol{U}),
\end{aligned}
$$

where

$\boldsymbol{O}^{\mathrm{l}}\left(s, s^{\prime}\right) \equiv \boldsymbol{E} \otimes \boldsymbol{O}\left(s, s^{\prime}\right) ; \quad \boldsymbol{O}^{\mathrm{r}}\left(s, s^{\prime}\right) \equiv \boldsymbol{O}\left(s, s^{\prime}\right) \otimes \boldsymbol{E}$.

Equation (50) is the starting point for the calculation of the mean value of $\boldsymbol{Y}(0) \otimes \boldsymbol{Y}(0)$.

\subsection{Averaging second-order moments}

In this section we show that the average of $\boldsymbol{Y}(0) \otimes \boldsymbol{Y}(0)$ over all the realizations of the KAP can be written in the form

$\langle\boldsymbol{Y}(0) \otimes \boldsymbol{Y}(0)\rangle_{\mathrm{KA}}=B_{1}^{2} \boldsymbol{M}(\boldsymbol{U} \otimes \boldsymbol{U})$,

where $\mathcal{M}$ is a $16 \times 16$ matrix which can be written as

$\mathcal{M}=\mathcal{M}^{1}+\mathcal{M}^{\mathrm{r}}$,

with

$$
\begin{aligned}
\mathcal{M}^{1, \mathrm{r}}= & {\left[\mathcal{E}-v\left\langle\tilde{\boldsymbol{G}}_{\mathrm{S}}(v)\right\rangle\right]^{-1} } \\
& \times\left\langle\tilde{\mathcal{G}}_{\mathrm{S}}(v) \tilde{\boldsymbol{O}}_{\mathrm{S}}^{1, \mathrm{r}}(v)\right\rangle\left[\mathcal{E}-v\left\langle\tilde{\boldsymbol{O}}_{\mathrm{S}}^{1, \mathrm{r}}(v)\right\rangle\right]^{-1}, \\
\tilde{\boldsymbol{O}}_{\mathrm{S}}^{1}(v) \equiv & \boldsymbol{E} \otimes \tilde{\boldsymbol{O}}_{\mathrm{S}}(v), \quad \tilde{\boldsymbol{O}}_{\mathrm{S}}^{\mathrm{r}}(v) \equiv \tilde{\boldsymbol{O}}_{\mathrm{S}}(v) \otimes \boldsymbol{E},
\end{aligned}
$$

and $\tilde{\mathcal{G}}_{\mathrm{S}}(v)$ the Laplace transform of the static double Green's function. We recall that $\tilde{\boldsymbol{O}}_{\mathrm{S}}(v)$ is the Laplace transform for $p=v$ of the static propagation operator $\boldsymbol{O}\left(s, s^{\prime}\right)$ (see Eq. (12)). The explicit expressions of the Laplace transforms are (see Eq. (13))

$$
\tilde{\boldsymbol{O}}_{\mathrm{S}}(v)=[v \boldsymbol{E}+\boldsymbol{K}]^{-1} ; \quad \tilde{\boldsymbol{G}}_{\mathrm{S}}(v)=[v \mathcal{E}+\mathcal{K}]^{-1} .
$$

We now give a proof of Eq. (54) based on the summation of a series, the $N$ th term of the series corresponding to all possible realizations having $N$ jumping points.

\section{Proof}

Taking the average of Eq. (50), we see that

$$
\mathcal{M}^{1}=\left\langle\int_{0}^{\infty} \mathcal{G}(0, s) \int_{s}^{\infty} \tilde{O}^{1}\left(s, s^{\prime}\right) \mathrm{d} s^{\prime} \mathrm{d} s\right\rangle_{\mathrm{KA}},
$$

with a similar definition for $\mathcal{M}^{\mathrm{r}}$. To simplify the notation, we drop the superscript 1 on $\mathcal{M}^{1}$ and $\boldsymbol{O}^{1}$.

We now consider an interval $\left[0, s^{\prime}\right]$, and examine all the realizations of the KAP. We characterize them by the number of jumping points $N$ in the interval $\left[0, s^{\prime}\right]$. We stress that $s^{\prime}$ varies from $s$ to $\infty$, while $s$ varies from 0 to $\infty$. In Sect. 2.3 we have already introduced the elements needed here, namely that the probability to have no jump in an interval of length $L$ is $\mathrm{e}^{-v L}$ and that the probability to have a jump in a small interval $\delta s_{i}$ around $s_{i}$ is $v \delta s_{i}$. The proof is based on the remarks that $\mathcal{G}\left(s, s^{\prime}\right)$ and $\mathcal{O}\left(s, s^{\prime}\right)$ satisfy a semi-group property and that they can be replaced by their static values if there is no jumping points between $s$ and $s^{\prime}$.

For $\boldsymbol{N}=0$, we have no jump in $\left[0, s^{\prime}\right]$ hence no jump in $[0, s]$ and $\left[s, s^{\prime}\right]$, so we can replace $\mathcal{G}(0, s)$ and $\boldsymbol{O}\left(s, s^{\prime}\right)$ by $\mathcal{G}_{\mathrm{S}}(s)$ and $\boldsymbol{O}_{\mathrm{S}}\left(s^{\prime}-s\right)$, respectively. We can thus write

$$
\begin{aligned}
& \mathcal{M}_{0}= \\
& \left\langle\int_{0}^{\infty} \int_{s}^{\infty} \mathrm{e}^{-v s} \mathcal{G}_{\mathrm{S}}(s) \mathrm{e}^{-v\left(s^{\prime}-s\right)} \boldsymbol{O}_{\mathrm{S}}\left(s^{\prime}-s\right) \mathrm{d} s^{\prime} \mathrm{d} s\right\rangle,
\end{aligned}
$$

where the exponential term is the probability that there is no jump in the intervals $[0, s]$ and $\left[s, s^{\prime}\right]$ and the averaging over the random value of the vector magnetic field, i.e. over $P(\boldsymbol{H})$. The r.h.s. can be expressed in terms of the Laplace transforms of the static propagation operators. We thus obtain

$\mathcal{M}_{0}=\left\langle\tilde{\mathcal{G}}_{\mathrm{S}}(v) \tilde{\boldsymbol{O}}_{\mathrm{S}}(v)\right\rangle$,

where the average in the r.h.s. is over $P(\boldsymbol{H})$.

For $\boldsymbol{N}=1$, we have one jump, say at a point $s_{1}$, within an interval $\delta s_{1}$, which can lie in either one of the intervals $[0, s]$ or $\left[s, s^{\prime}\right]$. We consider the two cases separately.

Case (a): $0<s_{1}<s<s^{\prime}$

First we use the semi-group property to write

$\mathcal{G}(0, s) \boldsymbol{O}\left(s, s^{\prime}\right)=\boldsymbol{G}\left(0, s_{1}\right) \mathcal{G}\left(s_{1}, s\right) \boldsymbol{O}\left(s, s^{\prime}\right)$.

Since there is no jump in each of the intervals $\left[0, s_{1}\right],\left[s_{1}, s\right]$, $\left[s, s^{\prime}\right]$, we can replace the propagation operators by their static value, which depend only on the random value of the vector magnetic field $\boldsymbol{H}$. Now we remark that with the conditioning at $s_{1}$, the random values of $\boldsymbol{H}$ to the left and to the right of $s_{1}$ become independent. This implies that we can average separately over $\boldsymbol{P}(\boldsymbol{H})$ the factor $\boldsymbol{G}_{\mathrm{S}}\left(s_{1}\right)$ and the product $\boldsymbol{G}_{\mathrm{S}}\left(s-s_{1}\right) \boldsymbol{O}_{\mathrm{S}}\left(s^{\prime}-s\right)$. After averaging over all possible values of $s_{1}$, we thus obtain

$$
\begin{aligned}
\mathcal{M}_{1, a}= & \int_{0}^{\infty} \int_{0}^{s} \int_{s}^{\infty} v \mathrm{e}^{-v s_{1}}\left\langle\mathcal{G}_{\mathrm{S}}\left(s_{1}\right)\right\rangle \mathrm{e}^{-v\left(s-s_{1}\right)} \mathrm{e}^{-v\left(s^{\prime}-s\right)} \\
& \times\left\langle\mathcal{G}_{\mathrm{S}}\left(s-s_{1}\right) \boldsymbol{O}_{\mathrm{S}}\left(s^{\prime}-s\right)\right\rangle \mathrm{d} s^{\prime} \mathrm{d} s_{1} \mathrm{~d} s
\end{aligned}
$$

where the product of exponential terms, multiplied by $v$, is the probability of having only one jump at $s_{1}$ (within $\delta s_{1}$ ).

The integrations over $s_{1}, s$ and $s^{\prime}$ can be carried out explicitly in terms of the Laplace transforms $\tilde{\boldsymbol{G}}_{\mathrm{S}}(v)$ and $\tilde{\boldsymbol{O}}_{\mathrm{S}}(v)$. The integral over $s^{\prime}$ is already a Laplace transform. Changing the order of integration, the integral $\int_{0}^{\infty} \mathrm{d} s \int_{0}^{s} \ldots \mathrm{d} s_{1}$ can be transformed into $\int_{0}^{\infty} \mathrm{d} s_{1} \int_{s_{1}}^{\infty} \ldots \mathrm{d} s$. We thus obtain

$\boldsymbol{M}_{1, a}=v\left\langle\tilde{\mathcal{G}}_{\mathrm{S}}(v)\right\rangle\left\langle\tilde{\mathcal{G}}_{\mathrm{S}}(v) \tilde{\boldsymbol{O}}_{\mathrm{S}}(v)\right\rangle$

where the averages are over the distribution $P(\boldsymbol{H})$.

Case (b): $0<s<s_{1}<s^{\prime}$

Since $s_{1}$ is to the right of $s$, we now write

$\mathcal{G}(0, s) \boldsymbol{O}\left(s, s^{\prime}\right)=\boldsymbol{G}(0, s) \boldsymbol{O}\left(s, s_{1}\right) \boldsymbol{O}\left(s_{1}, s^{\prime}\right)$. 
Proceeding exactly as above, we obtain

$$
\begin{aligned}
\mathcal{M}_{1, b}= & \int_{0}^{\infty} \int_{s}^{\infty} \int_{s}^{s^{\prime}} \mathrm{e}^{-v s} \mathrm{e}^{-v\left(s_{1}-s\right)}\left\langle\mathcal{G}_{\mathrm{S}}(s) \boldsymbol{O}_{\mathrm{S}}\left(s_{1}-s\right)\right\rangle \\
& \times v \mathrm{e}^{-v\left(s^{\prime}-s_{1}\right)}\left\langle\boldsymbol{O}_{\mathrm{S}}\left(s^{\prime}-s_{1}\right)\right\rangle \mathrm{d} s_{1} \mathrm{~d} s^{\prime} \mathrm{d} s .
\end{aligned}
$$

Transforming the integral $\int_{s}^{\infty} \mathrm{d} s^{\prime} \int_{s}^{s^{\prime}} \ldots \mathrm{d} s_{1}$ into $\int_{s}^{\infty} \mathrm{d} s_{1} \times$ $\int_{s_{1}}^{\infty} \ldots \mathrm{d} s^{\prime}$, integrating over $s^{\prime}$, then over $s_{1}$ and finally over $s$, we obtain

$$
\mathcal{M}_{1, b}=v\left\langle\tilde{\mathcal{G}}_{\mathrm{S}}(v) \tilde{\boldsymbol{O}}_{\mathrm{S}}(v)\right\rangle\left\langle\tilde{\boldsymbol{O}}_{\mathbf{S}}(v)\right\rangle .
$$

For $N=2$, we have three different cases: (a) two jumping points, say $s_{1}$ and $s_{2}$, in the interval $[0, s]$ and zero in the interval $\left[s, s^{\prime}\right]$; (b) one jumping point $s_{1}$ in $[0, s]$ and one jumping point $s_{2}$ in $\left[s, s^{\prime}\right]$; (c) zero jumping points in $[0, s]$ and two jumping points in $\left[s, s^{\prime}\right]$. With the same kind of arguments as above, we obtain

$$
\begin{aligned}
& \mathcal{M}_{2, a}=v^{2}\left\langle\tilde{\boldsymbol{G}}_{\mathrm{S}}(v)\right\rangle^{2}\left\langle\tilde{\boldsymbol{G}}_{\mathrm{S}}(v) \tilde{\boldsymbol{O}}_{\mathrm{S}}(v)\right\rangle, \\
& \mathcal{M}_{2, b}=v^{2}\left\langle\tilde{\boldsymbol{G}}_{\mathrm{S}}(v)\right\rangle\left\langle\tilde{\boldsymbol{G}}_{\mathrm{S}}(v) \tilde{\boldsymbol{O}}_{\mathrm{S}}(v)\right\rangle\left\langle\tilde{\boldsymbol{O}}_{\mathrm{S}}(v)\right\rangle, \\
& \mathcal{M}_{2, c}=v^{2}\left\langle\tilde{\boldsymbol{G}}_{\mathrm{S}}(v) \tilde{\boldsymbol{O}}_{\mathrm{S}}(v)\right\rangle\left\langle\tilde{\boldsymbol{O}}_{\mathrm{S}}(v)\right\rangle^{2} .
\end{aligned}
$$

We can now construct the general formula for an arbitrary number of jumps. We denote by $s_{-}$the last jumping point before $s$ and by $s_{+}$the first jumping point after $s\left(s_{-}<\right.$ $\left.s<s_{+}\right)$. The two intervals $\left[s_{-}, s\right]$ and $\left[s, s_{+}\right]$will produce a term $\left\langle\tilde{\mathcal{G}}_{\mathrm{S}}(v) \tilde{\boldsymbol{O}}_{\mathrm{S}}(v)\right\rangle$. All the intervals to the right of $s_{+}$will contribute with factors $\left\langle\tilde{\boldsymbol{O}}_{\mathrm{S}}(v)\right\rangle$ and all the intervals to the left of $s_{-}$with factors $\left\langle\tilde{G}_{\mathrm{S}}(v)\right\rangle$. If the last jumping point $s_{N}$ is such that $s_{N}<s$, the term $\left\langle\tilde{\mathcal{G}}_{\mathrm{S}}(v) \tilde{\boldsymbol{O}}_{\mathrm{S}}(v)\right\rangle$ comes from the intervals $\left[s_{N}, s\right]$ and $[s, \infty]$ and if the first jumping point $s_{1}$ is such that $s<s_{1}$, then this term comes from the intervals $[0, s]$ and $\left[s, s_{1}\right]$.

Summing all the contributions from $N=0$ to infinity, we find the result given in Eq. (54) for the matrices $\mathcal{M}^{1}$ and $\mathcal{M}^{\mathrm{r}}$. The central term corresponds to the interval $\left[s_{-}, s_{+}\right]$, the term to its right contains the contributions of all the intervals to the right of $s_{+}$and the term to its left the contributions of all the intervals between 0 and $s_{-}$.

We can now write an explicit expression for $\sigma_{i}^{2}(0)$. Since we have assumed that the line and continuum source functions are unpolarized, $\boldsymbol{U}=[1,0,0,0]^{\mathrm{T}}$ and $\boldsymbol{U} \otimes \boldsymbol{U}=[1,0, \ldots, 0]^{\mathrm{T}}$. Hence, only the first column in the matrix $\mathcal{M}$ will contribute to $\langle\boldsymbol{Y}(0) \otimes \boldsymbol{Y}(0)\rangle_{\mathrm{KA}}$. For Stokes $I$ and $V$ we thus have

$\sigma_{1}^{2}(0)=B_{1}^{2}\left[\mathcal{M}(1,1)-\left[\langle\tilde{\boldsymbol{O}}(0,0)\rangle_{\mathrm{KA}}(1,1)\right]^{2}\right]$,

and

$\sigma_{4}^{2}(0)=B_{1}^{2}\left[\boldsymbol{M}(16,1)-\left[\langle\tilde{\boldsymbol{O}}(0,0)\rangle_{\mathrm{KA}}(4,1)\right]^{2}\right]$,

where the matrix $\langle\tilde{\boldsymbol{O}}(0,0)\rangle_{\mathrm{KA}}$ is given in Eq. (17) and the numbers refer to the matrix elements. We have similar expressions for the dispersion around the mean values of Stokes $Q$ and $U$. We note also that the knowledge of the elements of $\mathcal{M}$ gives access to the cross-correlations $\left[\left\langle I_{i}(0) I_{j}(0)\right\rangle_{\mathrm{KA}}-\left\langle I_{i}(0)\right\rangle_{\mathrm{KA}}\left\langle I_{j}(0)\right\rangle_{\mathrm{KA}}\right]$, $i \neq j$.

In the microturbulent and macroturbulent limits, the expressions for the dispersion of the Stokes parameters are simpler. In the microturbulent limit, the dispersion is simply zero since all the coefficients in the transfer equation are replaced by their mean values. One is actually dealing with a deterministic problem. In the macroturbulent limit the second order moments can be deduced from the Unno-Rachkovsky solution which leads to

$$
\boldsymbol{M}_{\text {macro }}=\left\langle\boldsymbol{K}^{-1} \otimes \boldsymbol{K}^{-1}\right\rangle \text {. }
$$

One can check that Eq. (54) with $v=0$ is consistent with this expression. The macroturbulent limit is interesting because it provides an upper limit for the dispersion. This point is illustrated in the next section.

We checked the result given in Eqs. (52) to (56) by applying our summation method to a scalar transfer equation where the propagation matrix $\boldsymbol{K}$ is replaced by an absorption coefficient $K$. For this scalar problem, the second-order moment can also be calculated with a method introduced by Bourret et al. (1973) which relies on the introduction of new quadratic dependent variables, chosen in such a way that they satisfy a homogeneous system of linear stochastic equations. This method, restricted to scalar problems, has been applied by Auvergne et al. (1973) for the broadening of spectral lines by a turbulent velocity field.

Once the problem of calculating the second-order moments of $\left\langle I_{i}(0) I_{j}(0)\right\rangle_{\mathrm{KA}}$ has been reduced to the calculation of the mean value of the r.h.s. in Eq. (50), it is very likely that methods somewhat different from the summation method presented here can be set up. In particular L94 method should work, although it could be algebraically somewhat cumbersome since it does not make direct use of the Laplace transform of the evolution operator.

\subsection{Numerical evaluation of the dispersion}

To calculate the dispersion of the Stokes parameters we must evaluate the elements of the matrix $\mathcal{M}$. The averages over $P(\boldsymbol{H})$ (see Eq. (54)) are performed with Gauss-Legendre quadratures. The integration over the magnetic field intensity can be carried out with the same grid points as for the calculation of the mean Stokes parameters (see Sect. 3.2). The angular integrations over the polar angles $\theta$ and $\phi$ require more refined grids. Typically one needs around 30 points to calculate the dispersion while 10 or less are enough for the mean values. We note also that the width $x_{\max }$ of the frequency domain must be significantly increased.

In the macroturbulent limit, the calculation of the dispersion is much simpler since

$\sigma_{i}^{2}(0)_{\text {macro }}=\left\langle I_{i}^{2}(0)\right\rangle-\left\langle I_{i}(0)\right\rangle^{2}$,

where $I_{i}(0)$ is the Unno-Rachkovsky solution for the Stokes parameter $I_{i}$.

We note also that all the results obtained for the second-order moments of the Stokes parameters hold for the residual Stokes parameters, provided we divide them by $B_{1}^{2}$ (see Eq. (52)).

Figure 10 shows $\left\langle r_{X}\right\rangle \pm \sigma_{X},\left(\sigma_{X}>0\right)$ for the four Stokes parameters and different values of $v$ (we use the subscript the same convention as in Sect. 3.2). The magnetic field parameters are $\Delta H_{0}=1.0$ and $\gamma_{\mathrm{H}}=1$ as in Figs. 4 and 5 and hence correspond to a case of moderately strong fluctuations $(f=1)$. The direction of the mean field is $\theta_{0}=45^{\circ}$ and $\phi_{0}=30^{\circ}$. Comparing with Figs. 4 and 5 where $\phi_{0}=0^{\circ}$ and $\theta_{0}=0^{\circ}$ or $\theta_{0}=90^{\circ}$, we see that $\left\langle r_{Q}\right\rangle$ has become much smaller, as expected, and has become almost insensitive to the value of $v$ (on the scale of Fig. 10). Of course, $\left\langle r_{V}\right\rangle$ has also become somewhat smaller and remains almost independent of $v$. For $\left\langle r_{I}\right\rangle$, the dependence on $v$ does seem to depend on the direction of the mean field. 


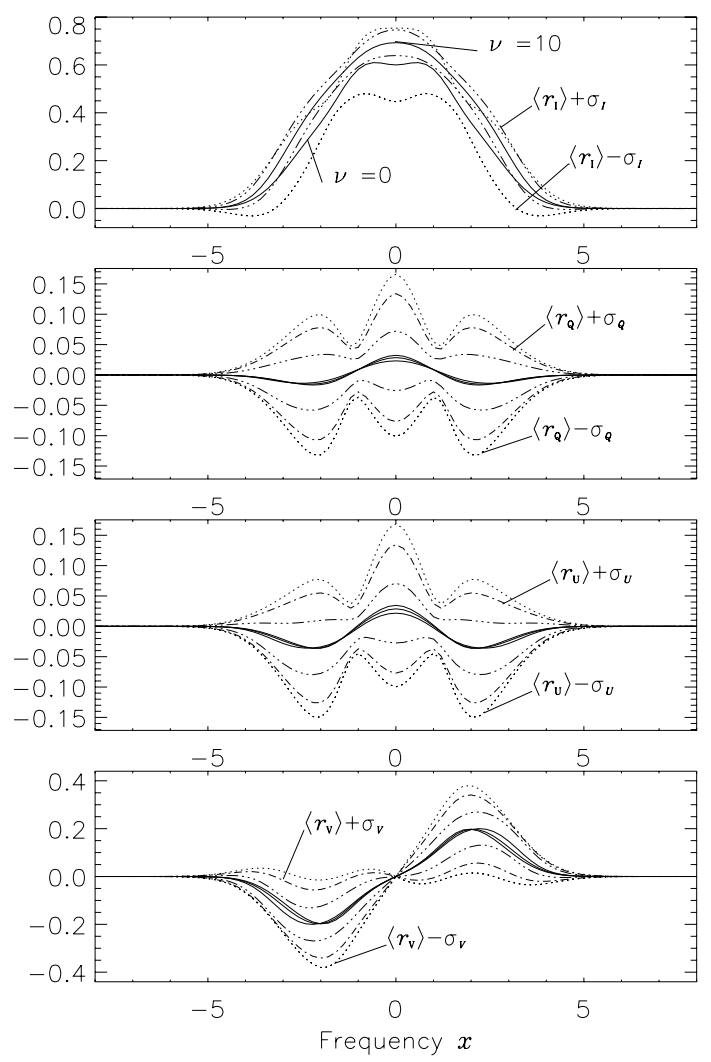

Fig. 10. Mean Stokes parameters with dispersion for moderately strong fluctuations of the magnetic field. The model parameters are: $\beta=10$, $\Delta H_{0}=1.0, \gamma_{\mathrm{H}}=1$. The direction of the mean magnetic field $\boldsymbol{H}_{0}$ is defined by $\theta_{0}=45^{\circ}$ and $\phi_{0}=30^{\circ}$. The full lines show the mean profiles and the discontinuous lines the mean values plus and minus the square root of the dispersion. The line types are: dotted $(v=0)$; dot-dashed $(v=1)$; triple-dot-dashed $(v=10)$. In this figure $\langle\ldots\rangle$ stands for $\langle\ldots\rangle_{\mathrm{KA}}$.

In contrast with the mean values, we see that the $\sigma_{X}$ are very sensitive to the value of $v$. They have their largest values in the macroturbulent limit $(v=0)$ and go to zero in the microturbulent limit. In the macroturbulent limit, the dispersion is quite large compared to the mean value. For the mean Stokes profiles, we have seen that the microturbulent limit is essentially reached when $v \simeq \beta$. Figure 10 shows that the dispersion has still a significant value when $v \simeq \beta$. This makes the dispersion much more sensitive to the characteristic scale of the random magnetic field.

Figure 11 shows the macroturbulent limit of $\sigma_{X}$ calculated with Eq. (70) for $\Delta H_{0}=1.0$ (as in Fig. 10) and different values of $f$ varying between 0.5 and 4. For Stokes $I, Q$ and $U$ the dispersion has maxima at line center and at the frequencies corresponding to the inflexion points in the Stokes $I$ profile. For Stokes $V$, the dispersion is zero at line center for symmetry reason, and has its maximum at the inflexion points of $I$ also. The minima of $\sigma_{Q}$ and $\sigma_{U}$ correspond to the zero-crossing frequencies in the mean Stokes profiles.

Starting from a case of weak fluctuations $(f=0.5)$, we observe that the dispersion increases with $f$, as expected, until say $f=2$. For larger values of $f$, we observe a decrease of the peak value in the wings of $\sigma_{Q}, \sigma_{U}$ and $\sigma_{V}$, associated to a significant broadening which reflects the fact that the stronger the fluctuations of the magnetic field, the further out from line center can they be felt. At line center $\sigma_{Q}$ and $\sigma_{U}$ keep increasing with $f$, even beyond $f=2$. Numerical experiments, not presented, here indicate that $\sigma_{Q}$ and $\sigma_{U}$ saturate to a value around 0.25 but that this phenomenon is related to the choice of $P(\boldsymbol{H})$. When the

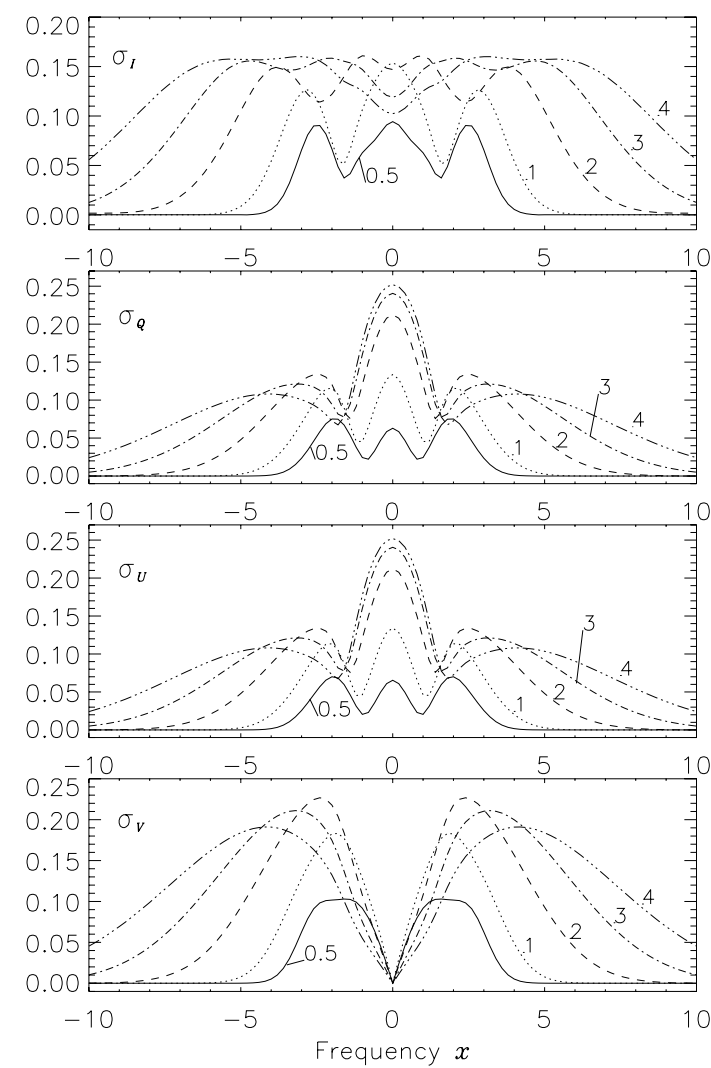

Fig. 11. Variation of the dispersion with the strength of the magnetic field fluctuations. The dispersion is shown only for the macroturbulent limit $v=0$. The line strength $\beta$ and the mean magnetic field parameters are the same as in Fig. 10. The curves are labeled with the value of $f$.

random magnetic field has a fixed direction and varies in intensity only, the values of $\sigma_{Q}$ and $\sigma_{U}$ at line center will decrease after going through a maximum. For Stokes $I$, the dispersion has a fairly complicated behavior, specially around the line center. The initial increase is also followed by some kind of saturation, but again, this is related to the choice of $P(\boldsymbol{H})$. In the wings, the behavior is essentially the same as for the other Stokes parameters. A more detailed analysis of the dispersion is deferred to a subsequent paper.

\section{Various extensions}

When some of the assumptions that were introduced to obtain explicit expressions for the mean Stokes parameters are dropped, it may still be possible to write an integral equation for the mean propagation operator. In some cases this equation can still be solved explicitly by a Laplace transform method, but in general a numerical solution is required. A few examples are given below.

\subsection{Exponential source function}

It follows from the solution of Eq. (1) (see Eq. (A.11)) that the mean value of the Stokes vector at the surface can be written as

$\langle\boldsymbol{I}(0)\rangle_{\mathrm{KA}}=\boldsymbol{S}(0)+\int_{0}^{\infty}\langle\boldsymbol{O}(0, s)\rangle_{\mathrm{KA}}\left[\frac{\mathrm{d} \boldsymbol{S}(s)}{\mathrm{d} s}\right] \mathrm{d} s$.

When $S$ is linear in $s$ one recovers Eq. (4). When $S$ has an exponential variation $\mathrm{e}^{-\alpha s}$ with $\alpha$ a constant, $\langle\boldsymbol{I}(0)\rangle_{\mathrm{KA}}$ can be 
expressed in terms of the Laplace transform of $\langle\boldsymbol{O}(0, s)\rangle_{\mathrm{KA}}$ for $p=\alpha$.

Let us consider an example presented in LL04 (p. 419) in which the continuum and line source functions are different and both have exponential terms. The transfer equation is now of the form

$$
\frac{\mathrm{d} \boldsymbol{I}}{\mathrm{d} \tau_{\mathrm{c}}}=[\boldsymbol{E}+\beta \boldsymbol{\Phi}] \boldsymbol{I}-\left[\boldsymbol{E} S_{\mathrm{c}}+\beta \boldsymbol{\Phi} S_{1}\right] \boldsymbol{U}
$$

The line and continuum source functions $S_{1}$ and $S_{\mathrm{c}}$ are given by

$$
\begin{aligned}
& S_{\mathrm{c}}\left(\tau_{\mathrm{c}}\right)=B_{0}+B_{1} \tau_{\mathrm{c}}+A_{1} \mathrm{e}^{-\alpha_{1} \tau_{\mathrm{c}}}, \\
& S_{1}\left(\tau_{\mathrm{c}}\right)=B_{0}+B_{1} \tau_{\mathrm{c}}+A_{1} \mathrm{e}^{-\alpha_{1} \tau_{\mathrm{c}}}-A_{2} \mathrm{e}^{-\alpha_{2} \tau_{\mathrm{c}}} .
\end{aligned}
$$

The term $A_{1} \mathrm{e}^{-\alpha_{1} \tau_{c}}$ can describe a chromospheric rise of temperature and the term $A_{2} \mathrm{e}^{-\alpha_{2} \tau_{c}}$ allows for a drop of the line source function below the continuum source function at optical depths $\tau_{\mathrm{c}} \leq 1 / \alpha_{2}$. Simple algebra (see also LL04) yields for the mean Stokes vector

$$
\begin{aligned}
& \langle\boldsymbol{I}(0)\rangle_{\mathrm{KA}}=\left[S_{1}(0) \boldsymbol{E}+B_{1}\langle\tilde{\boldsymbol{O}}(0,0)\rangle_{\mathrm{KA}}\right. \\
& \left.-\alpha_{1} A_{1}\left\langle\tilde{\boldsymbol{O}}\left(0, \alpha_{1}\right)\right\rangle_{\mathrm{KA}}+A_{2}\left(1+\alpha_{2}\right)\left\langle\tilde{\boldsymbol{O}}\left(0, \alpha_{2}\right)\right\rangle_{\mathrm{KA}}\right] \boldsymbol{U},
\end{aligned}
$$

where $\tilde{\boldsymbol{O}}(0, p)$ is defined in Eq. (11) and its mean value in Eq. (15).

When $S$ contains an exponential, it does not seem possible to transform the original transfer equation into a new equation with a homogeneous source term and obtain with the method described in Sect. 4 an explicit expression for the dispersion around the mean Stokes parameters.

\subsection{Arbitrary depth-dependence of source function and line strength}

We now assume that the line and continuum source functions and the ratio $\beta=\kappa_{\mathrm{o}} / \kappa_{\mathrm{c}}$ (introduced in Sect. 2.6) can vary with optical depth, but not the Zeeman propagation matrix $\boldsymbol{\Phi}$. This implies that the Doppler width is taken constant. There is no hope to obtain an exact result for the mean Stokes parameters, however an expression given in Pecker \& Schatzman (1959) for the difference $I_{c}(0)-I(0)$, in the case of non-polarized transfer, could be a good starting point for their numerical calculation. For the polarized case, the expression given in the above reference becomes

$\boldsymbol{I}_{\mathrm{c}}(0)-\boldsymbol{I}(0)=\left[w(0) \boldsymbol{E}+\int_{0}^{\infty} w^{\prime}(s) \boldsymbol{O}(0, s) \mathrm{d} s\right] \boldsymbol{U}$.

Here $s$ is the continuum optical depth along the line of sight, $w^{\prime}(s)$ and $w(0)$ are the derivative and surface value of the function

$w(s)=\int_{s}^{\infty} S_{\mathrm{c}}\left(s^{\prime}\right) \mathrm{e}^{-s^{\prime}} \mathrm{d} s^{\prime}-S_{1}(s) \mathrm{e}^{-s}$,

and

$\boldsymbol{O}\left(s, s^{\prime}\right)=\exp \left[-\boldsymbol{\Phi} \int_{s}^{s^{\prime}} \beta\left(s^{\prime \prime}\right) \mathrm{d} s^{\prime \prime}\right]$.

The derivation of Eq. (76) starts from the solutions of Eq. (72) for $\boldsymbol{I}_{\mathrm{c}}(0)$ and $\boldsymbol{I}(0)$. The main steps are the following: one combines the two terms containing $S_{\mathrm{c}}$ and introduces $\mathrm{d} I_{\mathrm{c}}^{+}(s) / \mathrm{d} s$ with $I_{\mathrm{c}}^{+}(s)=\int_{s}^{\infty} S_{\mathrm{c}}(s) \mathrm{e}^{-s} \mathrm{~d} s$. An integration by parts then yields Eq. (76).

The mean value $\langle\boldsymbol{O}(0, s)\rangle_{\mathrm{KA}}$ still satisfies Eq. (10) but the static propagation operator, as shown by Eq. (78), is now a function of $s$ and $s^{\prime}$.

\subsection{Depth-dependence of correlation length}

In the preceding sections, it has been assumed that $v$, the density of the Poisson distribution, is independent of the optical depth $s$ along the line of sight. If we let $v$ vary with depth, the Poisson process becomes a non-homogeneous Poisson process $^{1}$. The probability that no jumps occur between $s$ and $s^{\prime}$ is $\exp \left[-\int_{s}^{s^{\prime}} v\left(s^{\prime \prime}\right) \mathrm{d} s^{\prime \prime}\right]$. Equation (10) becomes

$$
\begin{aligned}
& \langle\boldsymbol{O}(0, s)\rangle_{\mathrm{KA}}=\left\langle\boldsymbol{O}_{\mathrm{S}}(s)\right\rangle \mathrm{e}^{-\int_{0}^{s} v\left(s^{\prime}\right) \mathrm{d} s^{\prime}} \\
& \quad+\int_{0}^{s} v\left(s^{\prime}\right) \mathrm{e}^{-\int_{s}^{s^{\prime}} v\left(s^{\prime \prime}\right) \mathrm{d} s^{\prime \prime}}\left\langle\boldsymbol{O}\left(0, s^{\prime}\right)\right\rangle_{\mathrm{KA}}\left\langle\boldsymbol{O}_{\mathrm{S}}\left(s-s^{\prime}\right)\right\rangle \mathrm{d} s^{\prime},
\end{aligned}
$$

with $\boldsymbol{O}_{\mathrm{S}}(s)$ still given by Eq. (5). This integral equation can only be solved numerically.

Some other generalizations can still lead to convolution equations for the mean evolution operator. For example, if $v$ depends on the modulus of the random magnetic field or if the random magnetic field consists of several fields with different characteristic scales. Such generalizations have been considered for the statistical Stark effect (Brissaud \& Frisch 1971).

\subsection{Arbitrary direction of propagation}

The results given in the previous sections hold for an outward directed ray normal to the surface of the atmosphere. They can easily be extended to the case of a ray making an angle $\theta$ with the vertical. It suffices to project on to the line of sight the quantities which describe the variations of the model along the normal to the atmosphere, such as the source function, absorption coefficients, and correlation length.

For the example treated in Sect. 5.1, $\langle\boldsymbol{I}(0, \mu)\rangle_{\mathrm{KA}}$ will be given by Eq. (75) with $B_{1}$ changed to $B_{1} \mu$ and $\alpha_{1}$ and $\alpha_{2}$ changed to $\mu \alpha_{1}$ and $\mu \alpha_{2}$, where $\mu=\cos \theta$. For the linear source function $S_{1}=S_{\mathrm{c}}=B_{0}+B_{1} \tau_{\mathrm{c}}$ treated in Sect. 2.6, the usual residual Stokes vector $\left(\boldsymbol{I}_{\mathrm{c}}(0, \mu)-\boldsymbol{I}(0, \mu)\right) / I_{\mathrm{c}}(0, \mu)$ will be given by Eq. (27) multiplied by $B_{1} \mu /\left(B_{0}+B_{1} \mu\right)$. For the example treated in Sect. 5.2, $w(s)$ and $\boldsymbol{O}\left(s, s^{\prime}\right)$ become

$w(s, \mu)=\int_{s}^{\infty} S_{\mathrm{c}}\left(s^{\prime}\right) \mathrm{e}^{-s^{\prime} / \mu} \frac{\mathrm{d} s^{\prime}}{\mu}-S_{1}(s) \mathrm{e}^{-s / \mu}$,

and

$\boldsymbol{O}\left(s, s^{\prime}, \mu\right)=\exp \left[-\boldsymbol{\Phi} \int_{s}^{s^{\prime}} \beta\left(s^{\prime \prime}\right) \frac{\mathrm{d} s^{\prime \prime}}{\mu}\right]$.

Here $s$ denotes the optical depth in the continuum in the direction normal to the atmosphere.

For the calculation of $\left\langle\boldsymbol{O}\left(s, s^{\prime}, \mu\right)\right\rangle$, the correlation length should also be projected along the line of sight, which means transforming $1 / v$ into $1 / \mu v$. Thus in Eqs. (27)-(29), $v$ should be changed to $v \mu$. This is also the change made in LL04 (see Eq. (9.280), p. 500), where $t_{\mathrm{e}}$, the mean length of the eddies measured in the vertical direction, becomes $t_{\mathrm{e}} / \mu$ along the line of sight. As a consequence, the more inclined with respect to the vertical are the rays, the closer is one to a macroturbulent type of averaging. This is consistent with a picture of random fluctuations organized in turbulent layers. Now, even in a plane parallel atmosphere, one may want to have a more or less isotropic distribution of turbulent eddies. This can be achieved by keeping the same value of $v$ (i.e. same correlation length) in all directions.

\footnotetext{
1 http://en.wikipedia.org/wiki/Non-homogeneous_ Poisson_process
} 


\section{Summary and concluding remarks}

This paper presents the first detailed investigation of the Zeeman effect created by a random magnetic field with a finite correlation length. The goal of this work is to overcome usual treatments whereby the correlation length of the magnetic field is either much smaller, or much larger, than a photon mean freepath, i.e. the microturbulent and macroturbulent limits. The random magnetic field is described by a Kubo-Anderson process which takes constant but random values on intervals of random length distributed according to a Poisson distribution of density $v$. The random magnetic field is thus characterized by a mean correlation length defined here as $1 / v$ and the probability distribution function $P(\boldsymbol{H})$ of the random values taken by the magnetic field. The micro and macroturbulent limits are recovered when the correlation length goes to zero or infinity.

The Kubo-Anderson process has been associated to a Milne-Eddington atmospheric model with a linear source function. This combination has allowed us to construct explicit expressions that were used to study numerically the mean Stokes parameters and their dispersion at the surface of the atmosphere. The main theoretical results concern the construction of:

(i) a convolution-type integral equation for the mean propagation operator associated to the Zeeman effect which can be solved explicitly for its Laplace transform;

(ii) an explicit expression for the mean Stokes parameters at the surface of the atmosphere which corroborates a result obtained by Landi Degl'Innocenti (1994);

(iii) an explicit expression for the second-order moments of the Stokes parameters which are needed to evaluate the dispersions and cross-correlations of Stokes parameters.

We have also given integral equations for the mean propagation operator when one relaxes some of the assumptions defining a Milne-Eddington model or Kubo-Anderson process, like depth-independent correlation length. These integral equations are not of the convolution type and must be solved numerically.

Numerical investigations have been carried out for a probability distribution function $P(\boldsymbol{H})$ describing a random magnetic field with mean value $\boldsymbol{H}_{0}$ and isotropic Gaussian fluctuations with dispersion $\sqrt{3} \sigma$. We have assumed a microturbulent velocity with a Gaussian distribution which is equivalent to incorporating an additional thermal broadening into the Doppler width of the line. In agreement with the Milne-Eddington model, the ratio $\beta=\kappa_{\mathrm{o}} / \kappa_{\mathrm{c}}$ of the line to continuum opacity has been taken constant. For weak lines ( $\beta$ order of unity or less), the Stokes parameters are essentially given by the profiles of the absorption coefficients and hence depend only on $P(\boldsymbol{H})$. For stronger lines, sensitive to the correlation length of the magnetic field, the mean Stokes parameters lie between the micro and macroturbulent limits. This is strictly true for Stokes $I$, because it is a positive quantity, and at line center for Stokes $Q$ and $U$. It is a bit more complicated for Stokes $V$ and the $\sigma$-components of Stokes $Q$ and $U$, because the position of the peaks depend on the correlation length. The microturbulent limit is reached when the correlation length is around unity in the line optical depth unit, i.e. when $\beta / v \simeq 1$.

The numerical calculations have been performed for $\beta=10$ (a few cases with $\beta=100$ have also been considered) for different values of the mean magnetic field $\boldsymbol{H}_{0}$, dispersion $\sigma$ and correlation length $1 / v$. The dispersion and mean field have been combined to construct a dimensionless parameter $f=\sqrt{2} \sigma / H_{0}$ which measures the relative strength of the magnetic field fluctuations. The assumption that the magnetic field fluctuations are isotropic influences some of the results but not the general trends which are summarized here.

Concerning the mean values, we have found that:

(i) for Stokes $I$, the variation between the micro and macroturbulent limits is between $10 \%$ and $20 \%$. It grows with the strength of the mean field $\boldsymbol{H}_{0}$ but seems fairly insensitive to value of $f$. Departures from the UR solution (Unno-Rachkovsky solution calculated with the mean field $\boldsymbol{H}_{0}$ ) can become quite large at line center when the $\sigma$-components are well separated, but this is partly due to the isotropy assumption;

(ii) Stokes $V$ shows very little dependence on the correlation length and hence, with reasonable confidence, may be calculated with the microturbulent limit. The departures from the UR solution are very large, unless $f$ is significantly smaller than unity;

(iii) for Stokes $Q$, the line center is quite sensitive to the correlation length of the magnetic field but only when $\boldsymbol{H}_{0}$ is in the transverse direction with respect to the line of sight, or close to it. For a given random magnetic field, the departures from the UR solution are larger for Stokes $Q$ than for Stokes $V$.

In sharp contrast with the mean Stokes parameters, dispersions around mean values are very sensitive to the correlation length and could probably serve as a diagnostic tool to determine the scale of unresolved features in the solar atmosphere. Dispersions have their maximum values in the macroturbulent limit, go to zero in the microturbulent limit and are very sensitive to the value of $f$. In relative value, the dispersion is smaller for Stokes $I$ than for the polarization components $Q, U$ and $V$.

In addition to the magnetic field, a whole set of other atmospheric random parameters (velocities, temperatures, densities, ...) are needed to properly describe a distribution of flux tubes or magnetohydrodynamic turbulence. These additional parameters should typically be described by the same type of random processes as the magnetic field, in particular the same correlation length. In this case all the theoretical results given here will hold, provided $P(\boldsymbol{H})$ is replaced by a joint distribution function $P\left(\boldsymbol{H}, \alpha_{1}, \alpha_{2}, \ldots\right)$, where the $\alpha_{i}$ stand for the other random parameters. If the random parameters have different correlation lengths, a KAP-type of modeling can still be set up. An example can be found in the case of the stochastic Stark effect (Brissaud \& Frisch 1971). A composite KAP is introduced to handle simultaneously the ion and electron electric fields with their quite different characteristic lengths due to the large mass difference between the two types of particles.

Acknowledgements. M.S. is financially supported by the Council of Scientific and Industrial Research (CSIR), through a Junior Research Fellowship (JRF Grant No: 9/890(01)/2004-EMR-I), which is gratefully acknowledged. M.S. is also grateful to the Indo-French Sandwich Thesis Program for making possible a visit to the Observatoire de la Côte d'Azur. Further K.N.N and M.S. are grateful to the Laboratoire Cassiopée (CNRS), the PNST (CNRS) and the French Ministère de l'Éducation Nationale for financial support during visits at the Observatoire de la Côte d'Azur where part of this work was completed. H.F. was supported by the Indo-French Center for the Promotion of Advanced Research (IFCPAR 2404-2) and by the Indian Institute of Astrophysics during her visits to Bangalore. She is grateful to J. Sánchez Almeida for stimulating discussions. The authors have also benefitted from constructive remarks from a referee.

\section{References}

Anderson, P. W. 1954, J. Phys. Soc. Japan, 9, 316

Auvergne, M., Frisch, H., Frisch, U., Froeschlé, Ch., \& Pouquet, A. 1973, A\&A, 29,93

Borrero, J. M., Lagg, A., Solanki, S. K., \& Collados, M. 2005, A\&A, 436, 333 
Bourret, R. C., Frisch, U., \& Pouquet, A. 1973, Physica, 65, 303

Brissaud, A., \& Frisch, U. 1971, J. Quant. Spectrosc. Radiat. Transf., 11, 1767

Brissaud, A., \& Frisch, U. 1974, J. Math. Phys., 15, 524

Carroll, T., \& Staude, J. 2003, in ASP Conf. Ser., 307, ed. J. Trujillo Bueno, \& J. Sánchez Almeida, 125

Carroll, T., \& Staude, J. 2005, Astron. Nachr., 326, 296

Childress, S., \& Gilbert, A. D. 1995, Stretch, Twist, Fold: The Fast Dynamo, Lecture Notes in Physics, 37 (Springer-Verlag)

Dolginov, A. Z., \& Pavlov, G. G. 1972, Soviet Ast., 16, 450 (transl. from Astron. Zhurnal 49, 555, 1972)

Domke, H., \& Pavlov, G. G. 1979, Ap\&SS, 66, 47

Faulstich, R. 1980, J. Quant. Spectrosc. Radiat. Transf., 24, 229

Frisch, H. 2006, A\&A, 446, 403

Frisch, H., \& Frisch, U. 1976, MNRAS, 175, 157

Frisch, H., Sampoorna, M., \& Nagendra, K. N. 2005, A\&A, 442, 11 (Paper I)

Frisch, H., Sampoorna, M., \& Nagendra, K. N. 2006, in Solar Polarization Proc. 4th SPW, ed. R. Casini, submitted

Frisch, U., \& Brissaud, A. 1971, J. Quant. Spectrosc. Radiat. Transf., 11, 1753

Froeschlé, Ch., \& Frisch, H. 1980, A\&A, 91, 202

Iyanaga, S., \& Kawada, Y. 1970, Encyclopedic Dictionary of Mathematics (Cambridge, Massachusetts: The MIT Press)

Jefferies, J., Lites, B. W., \& Skumanich, A. 1989, ApJ, 343, 920
Kubo, R. 1954, J. Phys. Soc. Japan, 9, 935

Landi Degl'Innocenti, E. 1987, in Numerical Radiative Transfer, ed. W. Kalkofen, 265

Landi Degl'Innocenti, E. 1994, in Solar Surface Magnetism, ed. R. J. Rutten, \& C. J. Schrijver, 29 (L94)

Landi Degl'Innocenti, E. 2003, in ASP Conf. Ser., 307, ed. J. Trujillo Bueno, \& J. Sánchez Almeida, 593

Landi Degl'Innocenti, E., \& Landolfi, M. 2004, Polarization in Spectral Lines (Kluwer Academic Publishers) (LL04)

Martinez Pillet, V. 2000, A\&A, 361, 734

Mihalas, D. 1978, Stellar Atmospheres, Second edn. (Freeman)

Pecker, J. C., \& Schatzman, E. 1959, Astrophysique Générale (Paris: Masson \& Cie)

Rees, D. 1987, in Numerical Radiative Transfer, ed. W. Kalkofen, 213

Sánchez Almeida, J. 1998, ApJ, 497, 967

Sánchez Almeida, J., Landi Degl'Innocenti, E., Martinez Pillet, V., \& Lites, B. W. 1996, ApJ, 466, 537

Sampoorna, M., Nagendra, K. N., \& Frisch, H. 2006, J. Quant. Spec. Radiat. Transf., in press

Silant'ev, N. A. 2005, A\&A, 433, 117

Stenflo, J. O. 1994, Solar Magnetic fields (Kluwer Academic Publishers) 
H. Frisch et al.: The Zeeman effect in a random medium, Online Material p 1

\section{Online Material}




\section{Appendix A: Some properties of the transport \\ operator}

For the benefit of the reader we recall here some of the main properties of the radiative transport operator for polarized transfer (Landi Degl'Innocenti 1987). The homogeneous transfer equation associated to Eq. (1) of the text may be written as

$\frac{\mathrm{d}}{\mathrm{d} s} \boldsymbol{I}(s)=\boldsymbol{K}(s) \boldsymbol{I}(s)$,

where $s$ is the ray-path coordinate which varies from 0 at the surface to $\infty$ in the interior, $\boldsymbol{K}$ the $4 \times 4$ propagation matrix and $\boldsymbol{I}$ the 4-dimensional Stokes vector. We consider rays propagating from infinity to the surface in the direction normal to the surface.

The Green's function, also called evolution or transport or propagation operator, is here defined by

$\boldsymbol{I}(s)=\boldsymbol{O}\left(s, s^{\prime}\right) \boldsymbol{I}\left(s^{\prime}\right)$,

with $s^{\prime}>s$ because photons propagate from the interior (positive values of $s$ ) to the surface at $s=0$. In Landi Degl'Innocenti (1987) and LL04, photons propagate from $-\infty$ to 0 , hence $\boldsymbol{O}\left(s, s^{\prime}\right)$ is defined with $s^{\prime}<s$ (as in time-dependent problems). The evolution operator obeys the limiting condition,

$\boldsymbol{O}(s, s)=\boldsymbol{E}$,

where $\boldsymbol{E}$ is the identity operator, and the semi-group property, which can be written as

$\boldsymbol{O}\left(s, s^{\prime}\right)=\boldsymbol{O}\left(s, s^{\prime \prime}\right) \boldsymbol{O}\left(s^{\prime \prime}, s^{\prime}\right), \quad s \leq s^{\prime \prime} \leq s^{\prime}$.

The evolution operator further satisfies two differential equations,

$\frac{\mathrm{d}}{\mathrm{d} s} \boldsymbol{O}\left(s, s^{\prime}\right)=\boldsymbol{K}(s) \boldsymbol{O}\left(s, s^{\prime}\right)$, and

$\frac{\mathrm{d}}{\mathrm{d} s^{\prime}} \boldsymbol{O}\left(s, s^{\prime}\right)=-\boldsymbol{O}\left(s, s^{\prime}\right) \boldsymbol{K}\left(s^{\prime}\right)$,

which can be derived from Eqs. (A.1) and (A.2) by taking the derivatives of Eq. (A.2) with respect to $s$ and $s^{\prime}$.

When the propagation matrix is a constant, the evolution operator is given by

$\boldsymbol{O}\left(s, s^{\prime}\right)=\exp \left[-\left(s^{\prime}-s\right) \boldsymbol{K}\right]$.

Using Eqs. (A.3) and (A.5) one can verify that the expression

$\boldsymbol{I}(s)=-\int_{s_{0}}^{s} \boldsymbol{O}\left(s, s^{\prime}\right) \boldsymbol{K}\left(s^{\prime}\right) \boldsymbol{S}\left(s^{\prime}\right) \mathrm{d} s^{\prime}+\boldsymbol{O}\left(s, s_{0}\right) \boldsymbol{I}\left(s_{0}\right)$,

where $\boldsymbol{I}\left(s_{0}\right)$ is the prescribed value of $\boldsymbol{I}$ at $s_{0}$, satisfies Eq. (1) of the text. Assuming that the source function increases less rapidly than an exponential at infinity, we obtain for the Stokes vector at the surface of a semi-infinite atmosphere,

$\boldsymbol{I}(0)=\int_{0}^{\infty} \boldsymbol{O}(0, s) \boldsymbol{K}(s) \boldsymbol{S}(s) \mathrm{d} s$

Using Eq. (A.6) we can rewrite this equation as

$\boldsymbol{I}(0)=-\int_{0}^{\infty}\left[\frac{\mathrm{d}}{\mathrm{d} s} \boldsymbol{O}(0, s)\right] \boldsymbol{S}(s) \mathrm{d} s$,

and after integrating by parts,

$\boldsymbol{I}(0)=\boldsymbol{S}(0)+\int_{0}^{\infty} \boldsymbol{O}(0, s)\left[\frac{\mathrm{d}}{\mathrm{d} s} \boldsymbol{S}(s)\right] \mathrm{d} s$.

When $\boldsymbol{S}(s)=\left(B_{0}+B_{1} s\right) \boldsymbol{U}$, we immediately obtain the result given in Eq. (3) of the text. 